\title{
Robust Object Categorization and Segmentation Motivated by Visual Contexts in the Human Visual System
}

\author{
Sungho Kim \\ Yeungnam University, 214-1 Dae-Dong Gyeongsan-Si, Gyeongsangbuk-Do, 712-749, Republic of Korea \\ Correspondence should be addressed to Sungho Kim, sunghokim@ynu.ac.kr \\ Received 7 April 2010; Accepted 9 November 2010 \\ Academic Editor: Steven McLaughlin
}

Copyright () 2011 Sungho Kim. This is an open access article distributed under the Creative Commons Attribution License, which permits unrestricted use, distribution, and reproduction in any medium, provided the original work is properly cited.

Categorizing visual elements is fundamentally important for autonomous mobile robots to get intelligence such as novel object learning and topological place recognition. The main difficulties of visual categorization are two folds: large internal and external variations caused by surface markings and background clutters, respectively. In this paper, we present a new object categorization method robust to surface markings and background clutters. Biologically motivated codebook selection method alleviates the surface marking problem. Introduction of visual context to the codebook approach can handle the background clutter issue. The visual contexts utilized are part-part context, part-whole context, and object-background context. The additional contribution is the proposition of a statistical optimization method, termed boosted MCMC, to incorporate the visual context in the codebook approach. In this framework, three kinds of contexts are incorporated. The object category label and figure-ground information are estimated to best describe input images. We experimentally validate the effectiveness and feasibility of object categorization in cluttered environments.

\section{Introduction}

Intelligent mobile robots should have visual perception capability akin to that provided by human eyes. Currently, many researchers have tried to develop human-like visual perception capabilities such as self-localization and object recognition for the intelligent mobile robots. Let us imagine that we have bought a new service robot and put it in our home environment. The robot should adapt to the strange environment automatically. It will wander the house and categorize each room as a kitchen, bath room, or living room. Additionally, it will categorize novel objects such as the door, sofa, TV, dining table, chair, or refrigerator. As we can see in this scenario, the two basic functions of an intelligent mobile robot are categorizing places and objects for automatic high-level learning about new environments. In addition, vision-based categorization system can be helpful for the visually handicapped people. Such system can give them useful place and object information. In the current stateof-the-art, topological localization remains at the level of image identification or matching to the same environment $[1,2]$. Object identification (recognition) of the same objects is almost matured due to the robustness of local invariant features such as SIFT and its generalized version, G-RIF $[3,4]$.

Currently, the categorization of general objects or scenes is an active research area in computer vision society to realize the helper robots and human assisting vision systems [5-7]. Therefore, many approaches have been proposed to handle object categorization. In general, the definition of object categorization is to assign a category label (normally basic level) for a novel object. The main difficulty of object categorization is the large intraclass variations. Among many sources of them, such as geometric shape variations and photometric color variations, textured appearances or surface markings are dominant in man-made objects as shown in Figure 1. Note the large variations of the surface markings at the interior regions of the objects. The effect of surface marking is much larger in man-made objects than in animals or plants due to creative design for beauty. These markings degrade the generalization capability of any categorization methods.

To our best knowledge, there has been few works published on the reduction of surface markings in object 


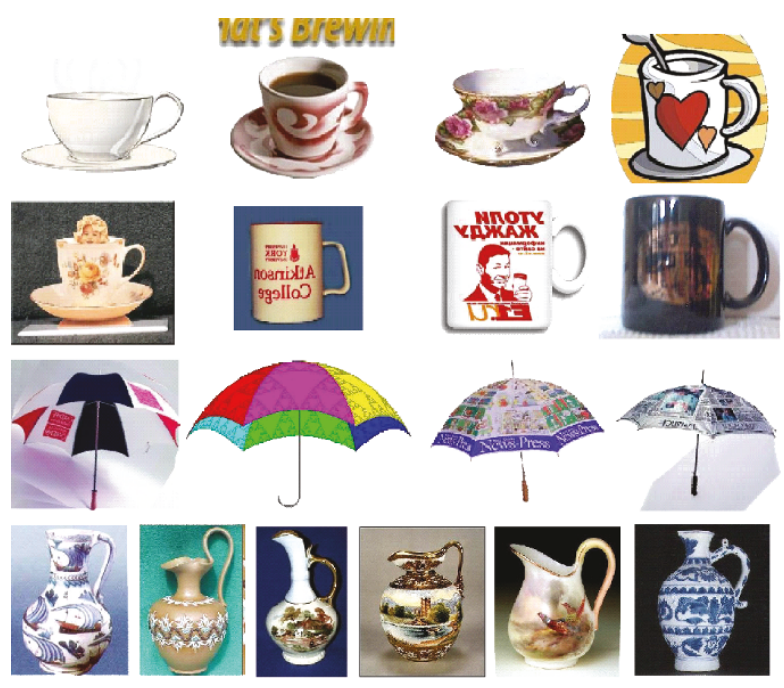

FIGURE 1: Examples of textured objects such as cups, umbrellas, and ewers (note the different surface markings).

categorization. Until now, most researchers have focused on how to minimize the intraclass variations caused by the object shape. We can categorize the current object representation schemes according to the relation of the geometric strength and intraclass variation as shown in Figure 2. As the strength of a geometric relation is weaker, the handling capability of intraclass variation is higher. At the same time, the discrimination power is reduced due to the weak spatial relation. Since the conventional principle component analysis (PCA) can represent whole objects with eigen vectors and eigen values, it is relatively weak to handle the geometric variations [8]. The constellation model of visual parts can handle geometric variations more flexibly $[5,9]$. It can handle visual variations with the part-based spring model. Flexible shape samples using geometric blur can represent large variations of shapes [10]. Bag of words, derived from document indexing, is a very robust method to visual variation because it considers no geometrical relations [11]. Texton, which is a more generalized version of bag of words, can categorize textured regions such as forest, sky, and sea [12]. A compromise of both extremes is the implicit shape model, which assigns pose information for each codebook [13].

Based on the bag of visual words, extended methods are proposed, such as spatial pyramid [14], hyperfeatures [15], and sparse localized features [16] that encode spatial information to histograms. Zhang et al. focused on classifier rather than feature extraction [17]. They combine nearest classifier with SVM, called SVM-KNN that shows upgraded performance for the Catech-101 DB (66.23\%). Varma and Ray proposed a domain-specific kernel learning method and obtained a classification rate of $79.85 \%$ for the same DB [18]. Perronnin et al. used universal codebooks and class specific codebooks that enhanced performance but required more memory space [19]. Wang proposed a discriminative codebook generation method by introducing multiresolution codebooks. This obtained superior discrimination compared to the single-resolution codebooks [20]. Yeh et al. presented an incremental method for learning a codebook in a dynamic environment, where images are continuously added to the database [21]. Gemert et al. introduced uncertainty (kernel density) modeling in a codebook that suffers less from the curse of dimensionality [22]. Zhang et al. proposed a learning method of multiple nonredundant codebooks for the categorization of complex objects that produced upgraded categorization performance [23]. However, those approaches do not consider the exterior variations such as the background clutter problem explicitly for optimal object categorization. These methods assume objects as whole images, so it is very similar to image classification.

If there is background clutter, the above approaches regard the clutter as parts of objects during learning. If we learn objects without background clutter and test two sets of images (segmented, cluttered) using the bag of visual words, we can obtain meaningful results as shown in Figure 3. These confusion matrices represent the object categorization for 48 man-made objects of Caltech DB. Note that categorization accuracy degrades from $90.13 \%$ to $60.97 \%$ (almost $30 \%$ ). Such experimental results are supported by the recent psychological experiment conducted by Grill-Spector and Kanwisher [24]. They showed that categorization and figureground segmentation are closely linked.

Several researchers have tried to reduce background clutter in object categorization. In the feature level, feature selection [25], or boosting [26] is proposed to overcome the clutter issue. Leibe et al. proposed combined object categorization and segmentation with an implicit shape model (ISM) [13, 27]. First they estimate object category and then segment the figure-ground pixel-wise. The spatial relation is modeled in a maximum entropy framework and leads to a high categorization rate [28]. Direct object region detection using a boundary fragment, a similar model to ISM, is also proposed. It shows some promising results to cluttered objects [29-31]. The partial matching method such as $\chi^{2}$ distance can alleviate background clutter during categorization using SVM [32]. Object segmentation with given category information using the random field model shows good segmentation results, even for occluded objects [33]. Shotton et al. proposed a multiclass object recognition and segmentation method based on jointly modeling texture, layout, and context [34]. Recently, Felzenszwalb et al. proposed an object detection system based on mixtures of multiscale deformable part model. It can detect deformable objects on challenging data [35].

All the approaches tried to solve the background clutter issue in terms of object categorization or object detection (localizing objects given a category). These methods are partial solutions to our goal, categorization and segmentation of unknown objects. Now, look at the Figure 4. Do you know what it is? This one figure motivates this research work. HVS can resolve what the object represents: it is a face. In this paper, our approach is motivated from several biological findings of human visual systems for the large intraclass variation and background clutter issues. The next section summarizes the mechanisms of the human visual system for visual object categorization in cluttered environments. 


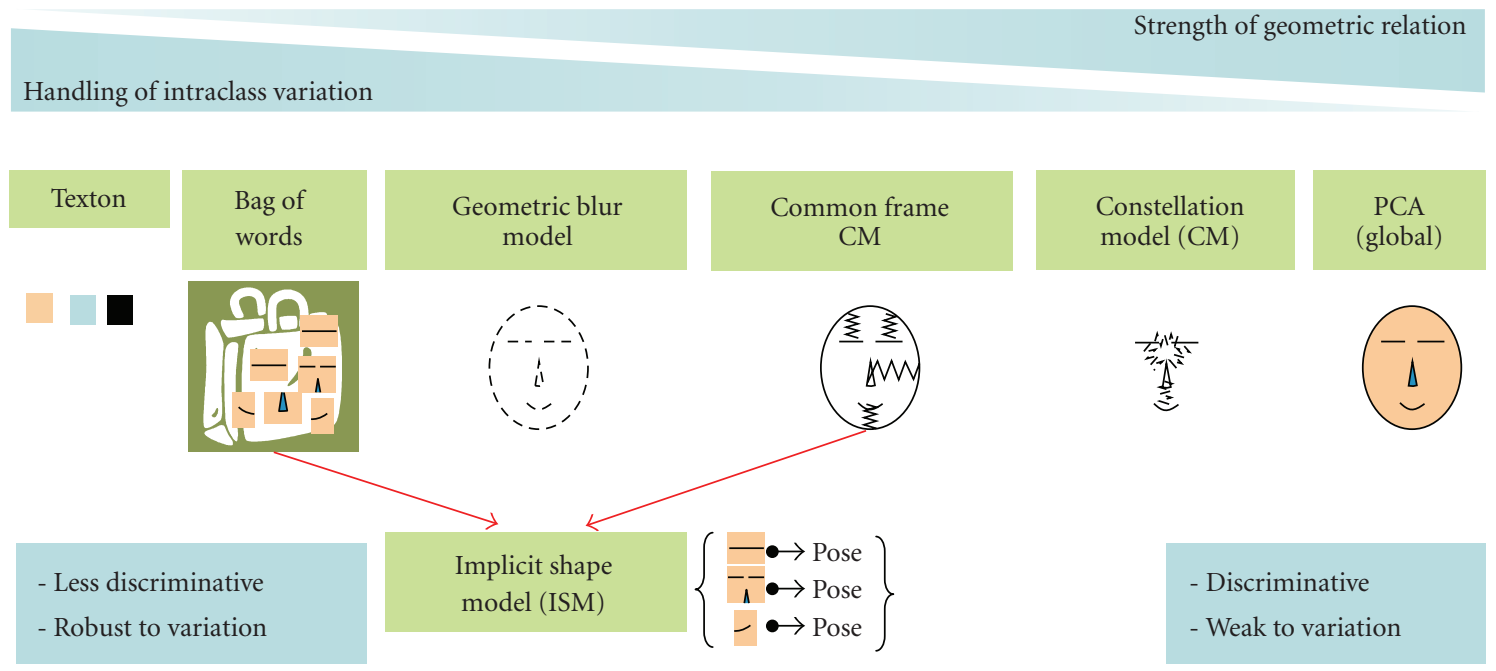

Figure 2: The trade off between handling capability of visual variation and object discriminability according to the different object representation schemes: Global PCA-based object representation uses strong pixel relation, which leads to strong discrimination but weak visual variation. Likewise, texton-based object representation discards pixel relation, which leads to weak discrimination but strong to visual variation.

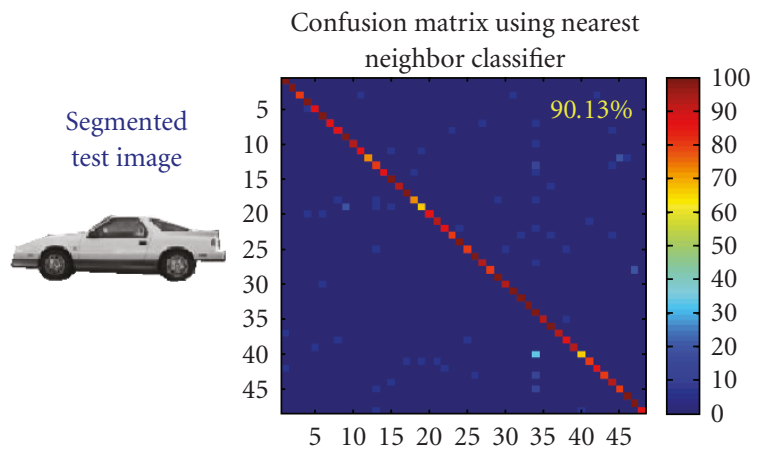

(a) Categorization results for segmented objects

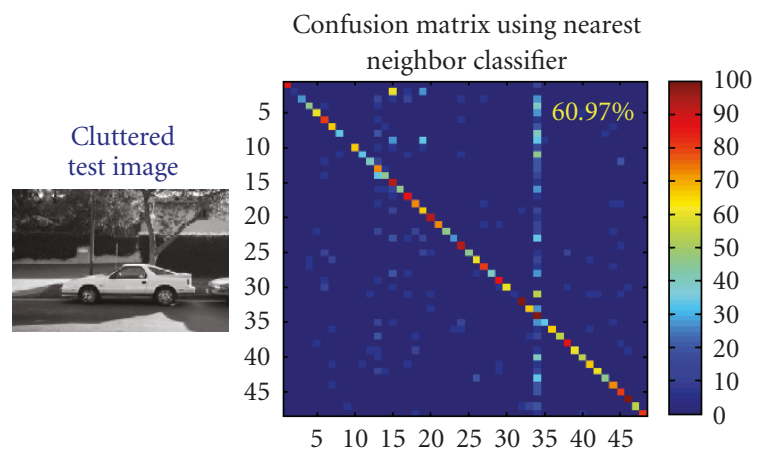

(b) Categorization results for cluttered objects

FIGURE 3: The effect of background clutter to object categorization using the bag of visual words. Confusion matrix measure is used for comparison.

\section{Visual Context in Human Visual System}

2.1. Part-Part Context. According to Gestalt's law, the human visual system actively utilizes the laws of proximity and similarity to discriminate the figural region and background region [36]. Proximity and similarity can group visual features into the figural region and background region. Visual context, such as part-part context, can be explained in terms of such Gestalt law. Part-part context means that parts belonging to the same object category should have the same property. Motivated from this psychological finding, we consider two properties of part relation: the same labeling and proximity, as shown in Figure 5. Parts belonging to an object share the same object labels. Furthermore, those parts are spatially very close. Gestalt's law of proximity and similarity for part-part context can provide a group of parts. Appropriate weights are assigned to those parts according to the probability of the same labeling and proximity. Contextually supported parts get stronger weights with a certain label. Parts belong to background region rarely show the clustering property compared to parts in the object region.

2.2. Part-Whole Context. Artale et al.'s research shows that the part-whole relation has been extensively used to convey structural information of objects [37]. Part information is used to predict whole object information (called transitivity property), such as hands in the human body and nose in the face. In addition, the interrelations among parts and whole can help us to recognize objects. Recent neurophysiological findings verified that visual recognition processes are hierarchical and interactively correlated through spike timing in the ventral visual stream [38]. Therefore, part information facilitates figure-ground, which also facilitates object categorization. At the same time, whole category information facilitates figure-ground segmentation that also facilitates part detection. Figure 6 represents the simple 


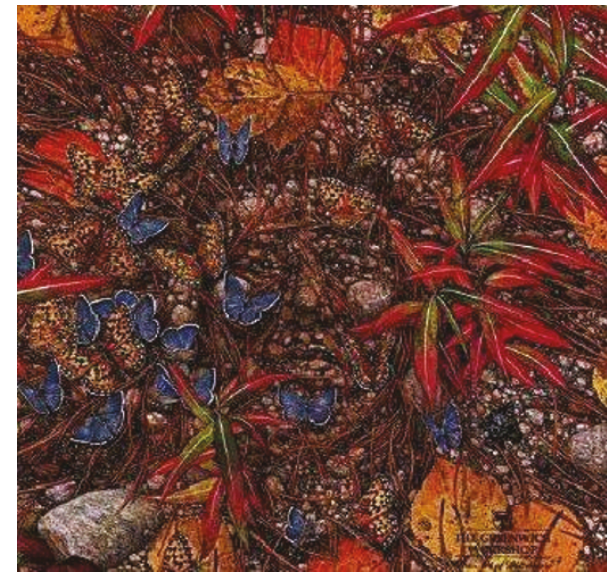

FIgURE 4: What is this? leaves or stones?
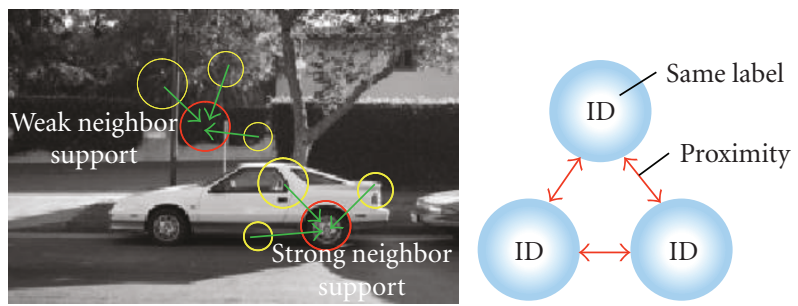

FIGURE 5: Similarity and proximity of part-part context.

concept of the part-whole relationship. Visual parts can predict the figure-ground and object center. Simultaneously, whole object category information can be used to verify recognition by carefully analyzing detected parts.

2.3. Object-Place Context. In addition to the part-part context, and part-whole context, the human visual system also utilizes object-place context [39]. In general, objects do not exist in a white background. Instead, objects exist in certain places, such as cars in a street, hair driers in a bathroom, and drills in a workshop. Therefore, object and place (background) are strongly correlated and usually coexist, as shown in Figure 7. If the relationship between object and place (background) is stronger, then we can categorize an unknown object more accurately.

These contexts are modeled by a directed graphical model that can provide object category with figure-ground segmentation. Bottom-up evidence from part-part context and part-whole context can provide the proposal function. Top-down generative inference using object-background context and whole-part context can provide the optimal category label, region of interest, and figure-ground mask that can best describe input features (both object and background features). The inference is conducted by multimodal MCMC sampling. Experimental results validate the power of the proposed framework for object categorization and figureground segmentation in a cluttered environment.

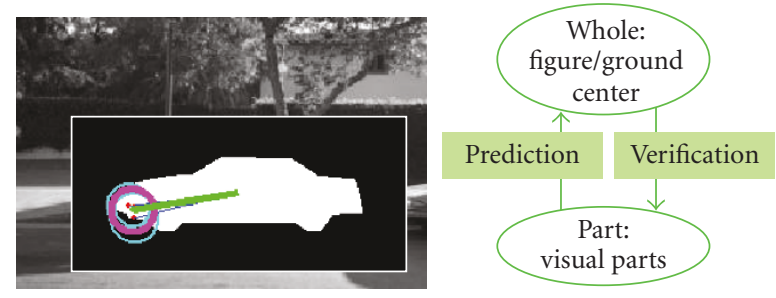

FIGURE 6: Part to whole prediction and whole to part verification in part-whole context.

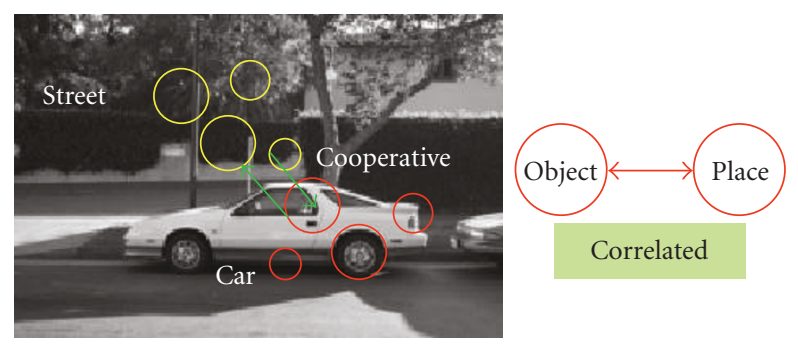

FIGURE 7: Strong correlation between object and background (place) context.

\section{Biologically Motivated Object Categorization}

3.1. Categorization Model of HVS. Conventionally, vision is considered to be accomplished by a feedforward chain of computations [40,41]. Serre et al. also introduce a hierarchical feedforward system that closely follows the organization of visual cortex and builds an increasingly complex and invariant feature representation by alternating between a template matching and a maximum pooling operation for object recognition [42]. Pinto et al. found that V1like model can recognize objects well [43]. However, recent neurophysiological experiments have provided a variety of evidence suggesting that feedback from higher-order areas (IT) can modulate the processing of the early visual cortex (V1, V2, V4) [38, 44-46]. A popular theory in the biological community to account for feedback is based on attention modulation and biased competition. From that perspective, visual processing is still primarily a series of feedforward computations, except that the computation and information flow are regulated by selective attention. Based on those neuropsychological findings, we can make a feasible object categorization model in the ventral visual pathway as shown in Figure 8. Along the ventral pathway, the specific visual properties and features to which cells are selective become more and more complex. See the left image in Figure 8. The first feature dimension extracted by the visual system in the retina and present in the LGN is luminance contrast. In the primary visual cortex, neurons use this input to build selectivity for line or edge orientation and sometimes display a certain degree of invariance to complex cells. Further down the line neurons respond to figure-ground boundaries in V2, and to complex geometric patterns in V4. Selectivity for the identity and category of complex objects or their components arises in the posterior part 
of the inferotemporal cortex (PIT) and is refined as visual information advances to the anterior part (AIT). Typically, neurons in IT respond to meaningful objects, in particular those with obvious biological relevance such as faces. IT is thus often considered as the end-point of the ventral stream hierarchy. This hierarchy is widely taken as evidence for a functional architecture in which, in a sequence of relatively small computational steps, visual areas extract from their afferents increasingly complex features of the stimulus theory. At the last levels, such features are by construction complex enough to represent object identity or category [38]. Note also that the visual processing modules such as, V1, V2, V4 are interrelated. Furthermore, each module has bottomup analysis and top-down synthesis for the correct image understanding.

The right image in Figure 8 is the corresponding visual processes implemented in this paper. Given an image, Gabor $90^{\circ}$ phase and Gabor $0^{\circ}$ phase images are obtained for corner and blob center detection. Simultaneously, edge map is detected for the object boundary points. These processes are performed in scale space pyramid. Such low level processing modules are similar to the V1 in HVS. Next, figure-ground segregation process exists like V2 in HVS. Dense local invariant structures extracted in V4, then final object categorization is performed on the top position. Those functional blocks interact with each other through bottomup analysis and top-down synthesis. Details will be explained in the following sections.

3.2. Object and Category Representation. To fully utilize the visual contexts, we propose a composite representation of object instance with region of interest (ROI, object center + scale), object boundary, and local parts, as shown in Figure 9. ROI represents the object center with the scale in this work. An object boundary or figure-ground mask divides an image into figural region and background region. Finally, local parts (clustered from dense features) represent the partbased object appearance. The ROI, figure-ground, and local parts are interrelated, like the spring model. In this joint model, local parts have an important role, since they relate ROI and the figure-ground boundary. That is, if we know a visual part, then we can predict ROI and object boundary. This is the part-whole context explained in the previous section. Every object instance is represented by ROI, Figureground mask, and codebook (including part appearance and pose).

We represent a category by extending the basic object representation model, as shown in Figure 10. There are universal appearance codebook and category-specific appearance codebook in the category representation. Local appearances of visual parts in the object instance are linked to category-specific codebook (CCB). Part pose information is stored in each part relative to the object center in the object instance. Category-specific codebooks are also linked to the universal codebook $(U C B)$ by comparing visual appearance. In Figure 10, wheels in the car codebook and in airplane codebook have a similar appearance. At the same time, each category also has a contextually related background codebook. Therefore, each category has a category-specific codebook and category-related background codebook. In addition, each UCB contains all possible link information to CCB. This link information is useful for bottom-up inference. Details of modeling and learning will be explained int the next sections.

\subsection{Mathematical Formulation for Object Categorization.} Look at the object in a cluttered environment, as shown in Figure 7 . We can generate such images if we have the category label, ROI (object center + scale), figure-ground mask, and codebook corresponding to input features belonging to the object category and category-related background. Figure 11(a) shows such an example of the generative procedure. We assume a single object in a cluttered background, since it is the basic block for multiple object categorization. The parameter $\{C, B\}$ represents a pair of category label $C$ and related background label $B$. Given a $\{C, B\}$, first we can generate the region of interest (ROI) of an object. ROI includes both object center and relative object scale. Therefore, the ROI parameter $V$ contains object center $\left(x_{c}, y_{c}\right)$ and object scale factor $(s)$ relative to model size. In the next layer, figureground mask $(M)$ is generated using the information of both category-background label and ROI. Mask $M$ is an array of $\{0,1\}$, where 0 represents the background pixel and 1 represents the foreground pixel. In the third layer, codebook index $F$ is selected using category-background information and figure-ground mask. The codebook index denotes label of category-specific codebook as shown in Figure 10. If the index belongs to the object region, our algorithm will search it from CCB and if it belongs to the background region, our algorithm will search it from the background codebook related to the CCB. Finally, we can generate input features $G$ using the selected codebook and ROI information. $G$ consists of a set of local appearance $A$ and part pose $X$ (total $N$ features). ROI information is reflected to part pose generation. Figure 11(b) shows the directed graphical model (Bayesian Net) exactly corresponding to Figure 11(a). White nodes represent hidden variables and shaded nodes represent observed variables. Note the causal relationship between nodes. Due to the $N$ input features, we replicate the codebook index and observation nodes $N$ times, as boxed regions. In addition to the top-down generative model, we draw bottom-up (dotted arrow) flow for fast estimation. This will be explained in the learning section.

Now, let us formulate the object categorization in cluttered images based on the directed graphical model. Given an unknown object with cluttered background, we can detect multiscale input features $G=\left\{g_{i}=\left(a_{i}, x_{i}\right)\right\}, i=1,2, \ldots, N$. $a_{i}$ denotes descriptor vector of local patch and $x_{i}$ denotes part position. Assume that we already have trained model $D$, which has labels, figure/ground masks, and ROIs with learned parameters (learning will be explained in the next section). Then, the object categorization and segmentation problem is to estimate the category label, $C$, figure-ground mask, $M(i, j)=1$ or 0 , and ROI, $V=\left\{x_{c}, y_{c}, s\right\}$. We set the solution vector as $H=(C, M, V)$ and the solution space as $\Omega$. Then the optimal solution can be represented by (1). 


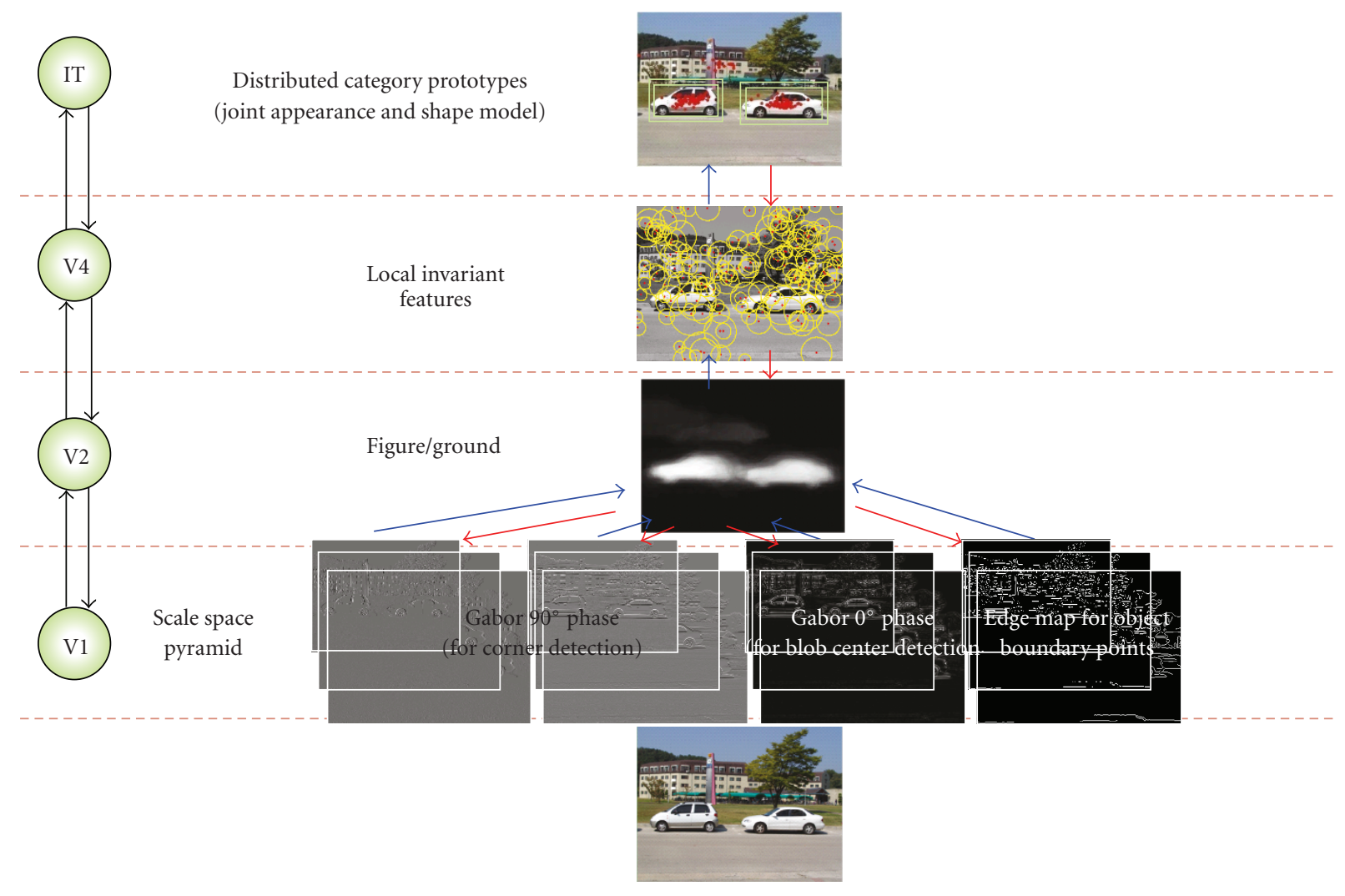

$\longrightarrow$ Bottom-up analysis

$\longrightarrow$ Top-down synthesis

FIGURE 8: The overall flow of object categorization of human visual system.

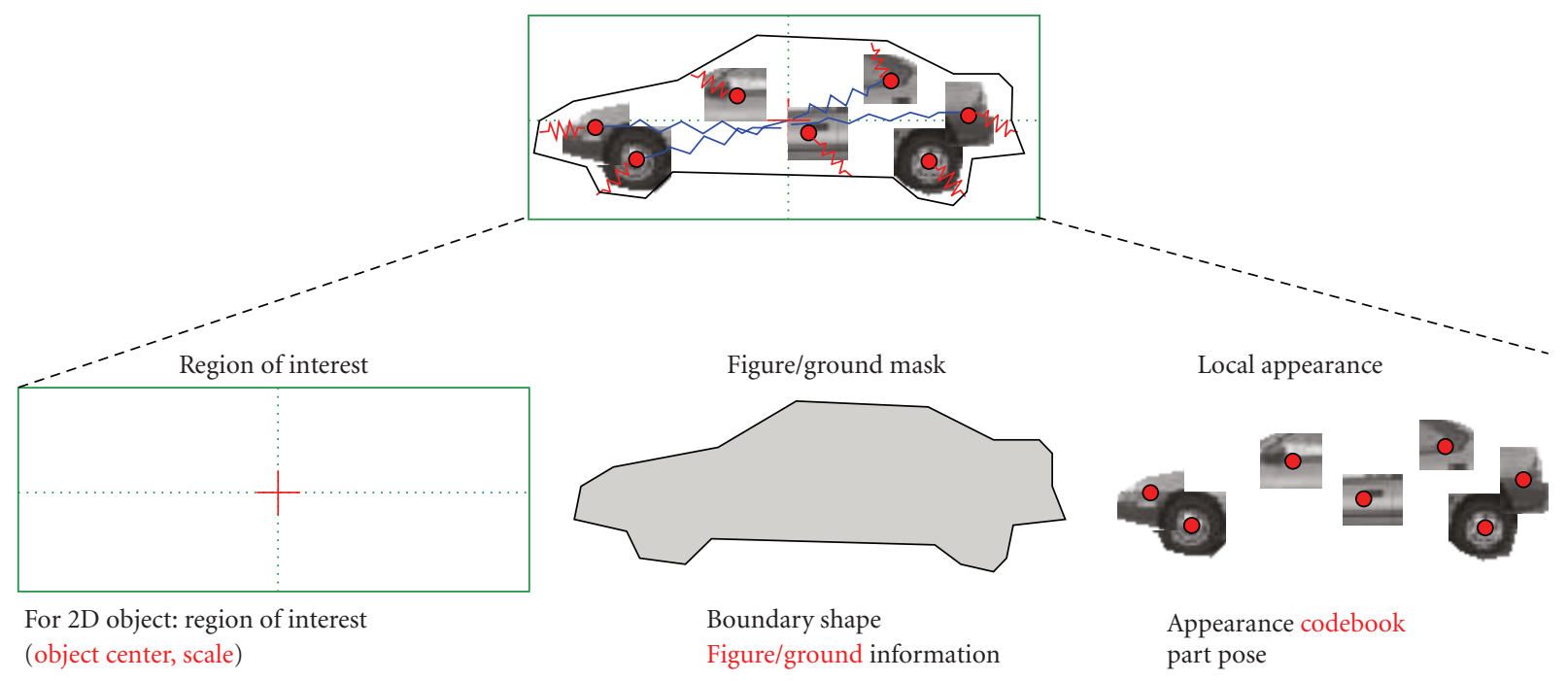

FIGURE 9: Basic representation of an object instance by region of interest (ROI), figure-ground mask, and local appearance.

Normalization is omitted for the simplicity, as we should maximize the posterior

$$
\begin{aligned}
H^{*} & =\arg \max _{H \in \Omega} p(H \mid G, D) \\
& =\arg \max _{H \in \Omega} p(H \mid H, D) p(H \mid D) .
\end{aligned}
$$

According to the directed graphical model (see Figure $11(\mathrm{~b}))$, the prior term $p(H \mid D)$ is decomposed into three conditional probabilites, as (2). If you want to know the basics of the graphical model, we recommend you see [47]. From trained data $D, p(C \mid D)$ represents the prior 


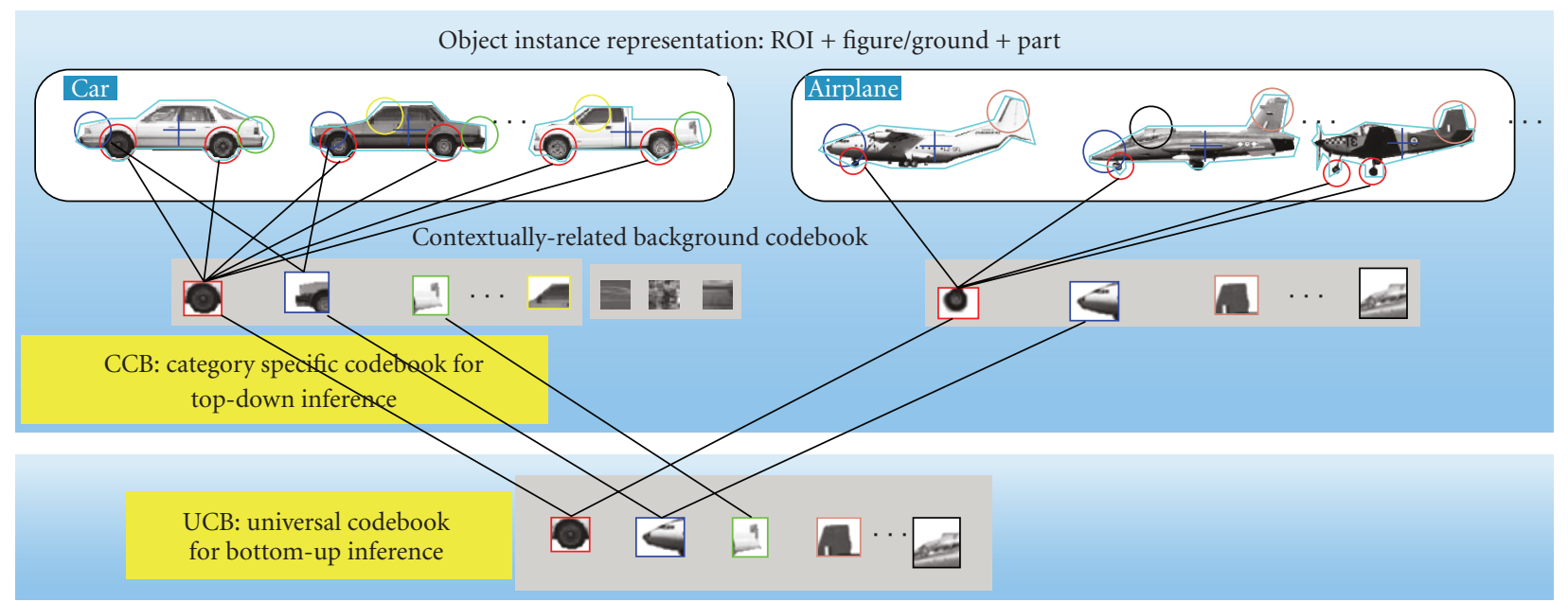

FIGURE 10: Category representation by two-layered codebook (universal codebook + category-specific codebook) with object instance representation.

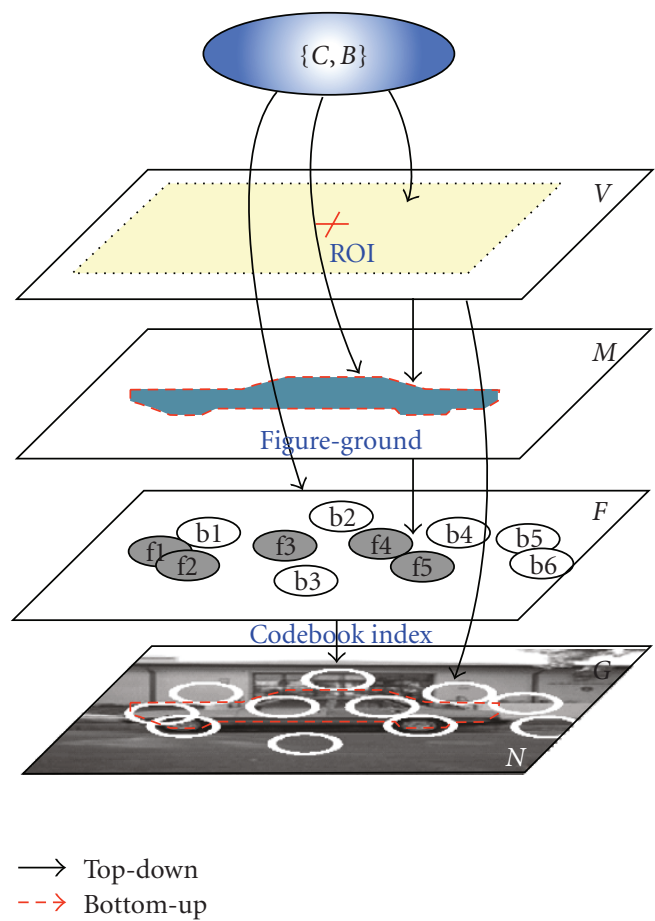

(a) Example of generative process

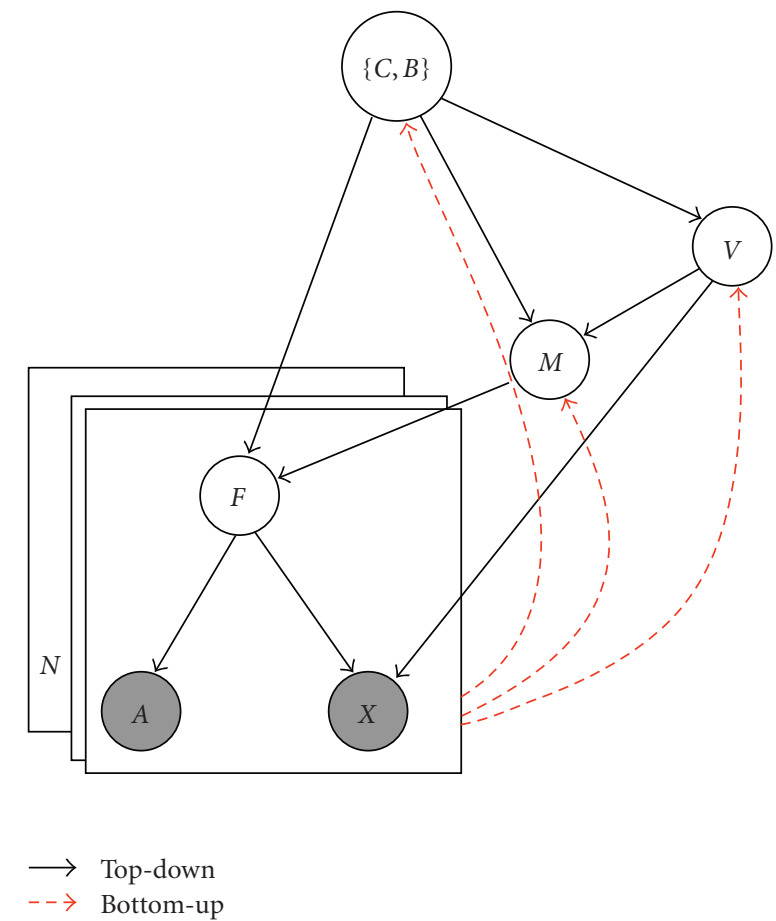

(b) Corresponding graphical model

FIGURE 11: (a) Generative framework for simultaneous object categorization and figure-ground segmentation in cluttered environment, (b) corresponding representation by directed graphical model (Bayesian Net).

of the category label. Given category label $C$ and $D, p(V \mid$ $C, D)$ represents the prior of ROI. Given a category, ROI with trained data, we can generate the figure-ground mask $M$ from $p(M \mid C, V, D)$

$$
p(H \mid D)=p(C \mid D) p(V \mid C, D) p(M \mid C, \mathrm{~V}, D)
$$

Given a hypothesis $H=(C, V, M)$ and trained data $D$, the likelihood term $p(G \mid H, D)$ is factorized as (3)

$$
p(G \mid H, D)=p_{f}\left(G_{f} \mid H, D\right) p_{b}\left(G_{b} \mid H, D\right),
$$

where $G_{f}=\left\{g_{m}: M\left(x_{m}\right)=1\right\}$ and $G_{b}=\left\{g_{n}: M\left(x_{n}\right)=\right.$ $0\}$. $G_{f}$ denotes the figural feature set and $G_{b}$ denotes the background feature set. In addition, $x_{m}$ is the position of 

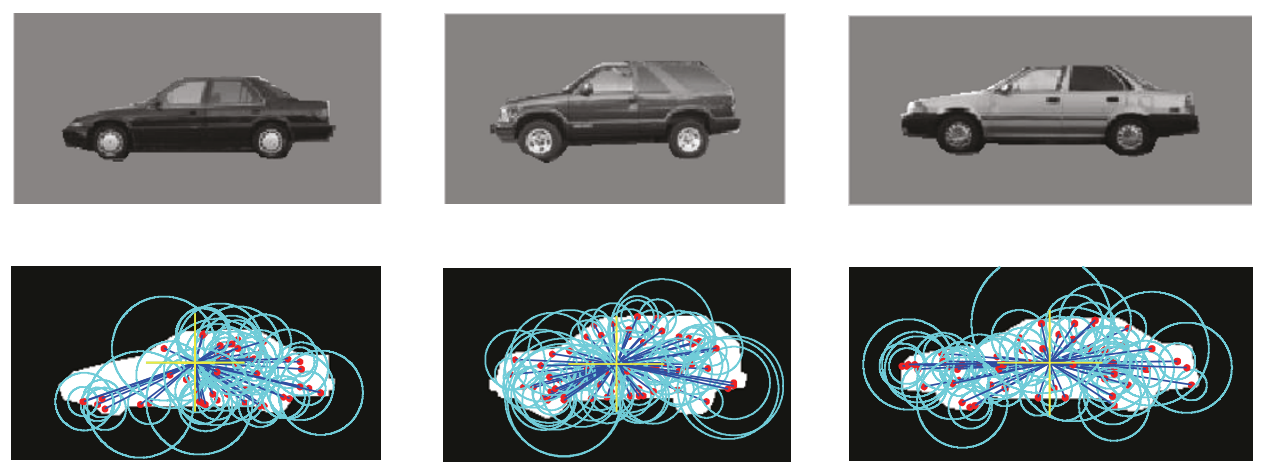

FIGURE 12: Foreground objects and detected local features.

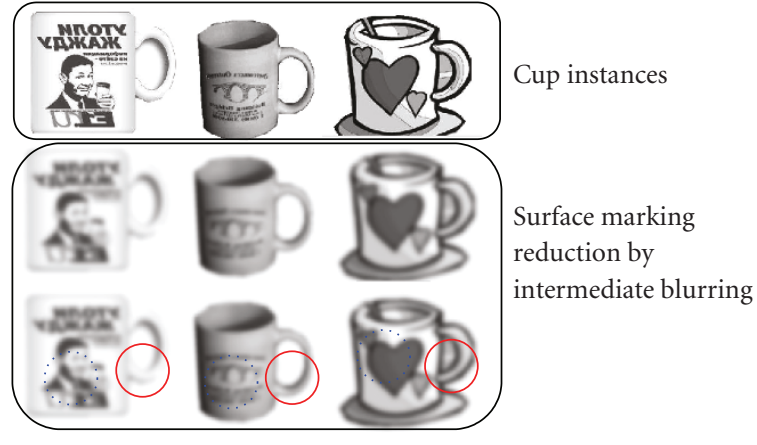

Repeatable part Surface marking part

FIGURE 13: Large intraclass variations due to surface markings and reduction strategy during codebook selection.

the input feature $g_{m}$ in the image space. If we assume $N$ independent input features, each likelihood term is defined as $(4)$

$$
\begin{aligned}
p_{f}\left(G_{f} \mid H, D\right)= & \prod_{i=1}^{N_{f}}\left(\sum_{j=1}^{\left|F_{f}\right|} \phi_{j} \mathcal{N}\left(a_{i} ; \mu_{a}^{j}, \Lambda_{a}^{j}\right)\right. \\
& \left.\cdot \mathcal{N}\left(x_{i} ; s \cdot \mu_{x}^{j}+\left(x_{c}, y_{c}\right), \Lambda_{x}^{j}\right)\right), \\
p_{b}\left(G_{b} \mid H, D\right)= & \prod_{i=1}^{N_{b}}\left(\sum_{j=1}^{\left|F_{b}\right|} \frac{\phi_{j} \mathcal{N}\left(a_{i} ; \mu_{a}^{j}, \Lambda_{a}^{j}\right)}{A}\right),
\end{aligned}
$$

where $N_{f}$ is the number of input features generated by the object codebook $F_{f}$ and $N_{b}$ is the number of input features generated by the background codebook $F_{b}$. Thus, $N_{f}+N_{b}=N$, the total number of input features. $\phi_{j}$ is the probability of codebook $j$. Foreground features are generated by Gaussian distributions $\mathcal{N}$ where $\mu_{a}^{j}$ and $\Lambda_{a}^{j}$ denote mean and covariance of appearance codebook $a_{i}$, respectively. $\mu_{x}^{j}$ denotes the average position of part $j$. Note that the codebook mean is affected by the ROI,
$V=\left(x_{c}, y_{c}, s\right)$. Background features are generated by the background codebook. However, the pose distribution is uniform, since they are distributed randomly in area $A$. Details of learning and inference will be explained in the next sections.

\section{Learning Parameters}

As shown in Figure 10, the category representation scheme consists of universal codebook and category-specific codebook. The category-specific codebook should be linked to the universal codebook. Each codeword is also linked to all similar parts in object instances. The learning items are first category-specific codebook, universal codebook, links between CCB and UCB; second, links between CCB and local patches in object instances that have ROI, figure/ground mask, and local patches. Note that training object instances are reused to handle large intraclass variations. The link information is a useful cue during bottom-up inference. From a scene feature, we can find similar UCB. Then, if we use the link information in the UCB, we can select the category-specific codebook. The links between CCB and local patches can give probable ROI, because each part has object center information. Finally, we introduce how to learn prior parameters, as shown in (2).

4.1. Step 1: Local Feature Extraction. First, we extract dense (or sparse) features, called G-RIF (Generalized Robust Invariant Feature), in scale-space from foreground object regions, as shown in Figure 12 [4]. G-RIF is similar to the well-known SIFT, but it is a generalized version of SIFT. It can detect corner-like interest points from a convolved image with $90^{\circ}$ phase of the Gabor kernel. It can also detect blob center points from a convolved image with $0^{\circ}$ phase of the Gabor kernel. In addition, we also use randomly sampled canny edge points, since this can enhance categorization capability in the codebook approach [48]. After interest point detection, the scale of local interest point is determined using the SIFT method. Then, the localized histogram of edge strength, orientation, hue makes a descriptor in GRIF. Positions $(x, y)$ of local features are defined in polar coordinates based on the object center to reflect object size changes. 


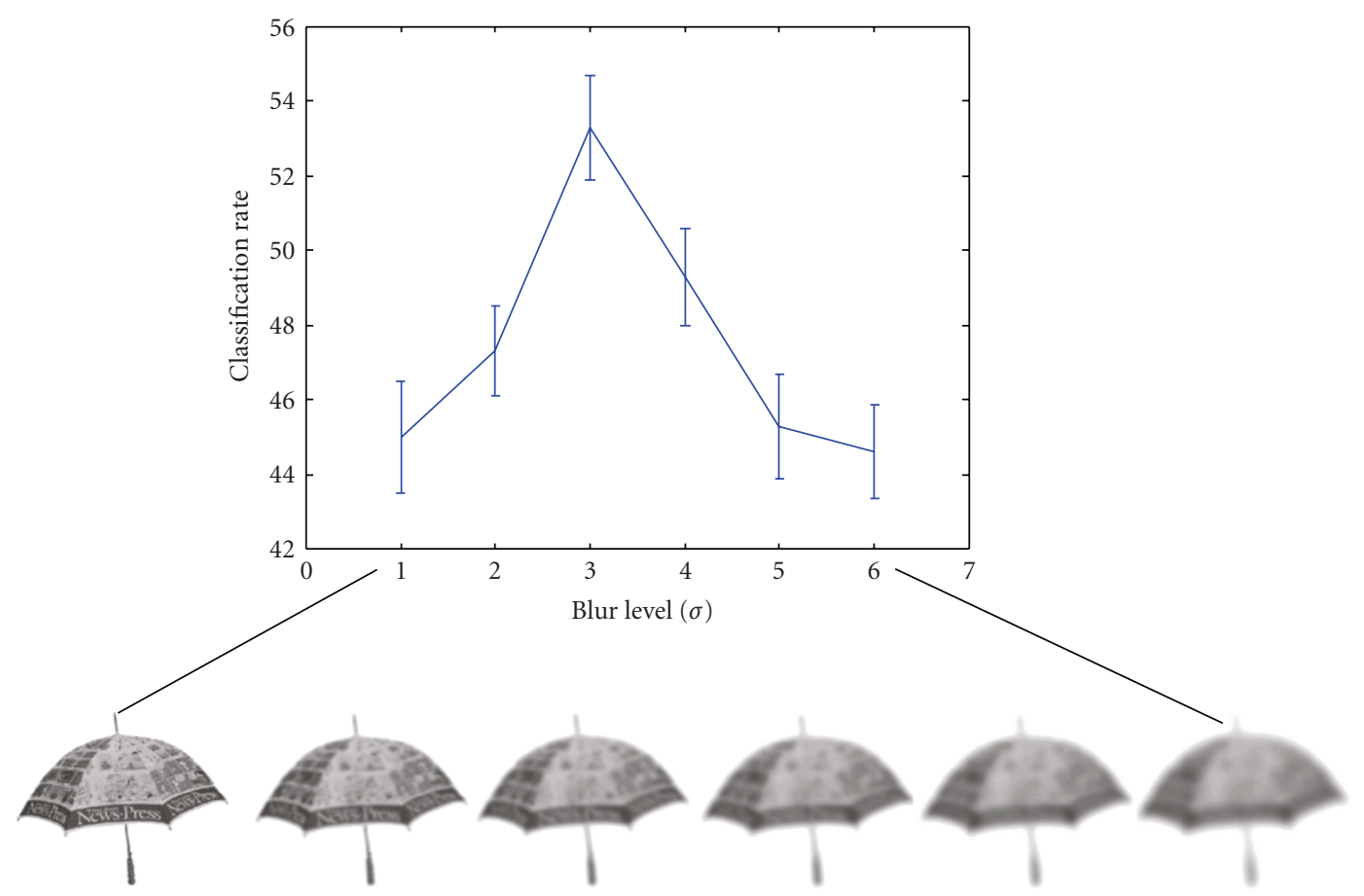

FIGURE 14: Evaluation of blurring level in terms of categorization rate.

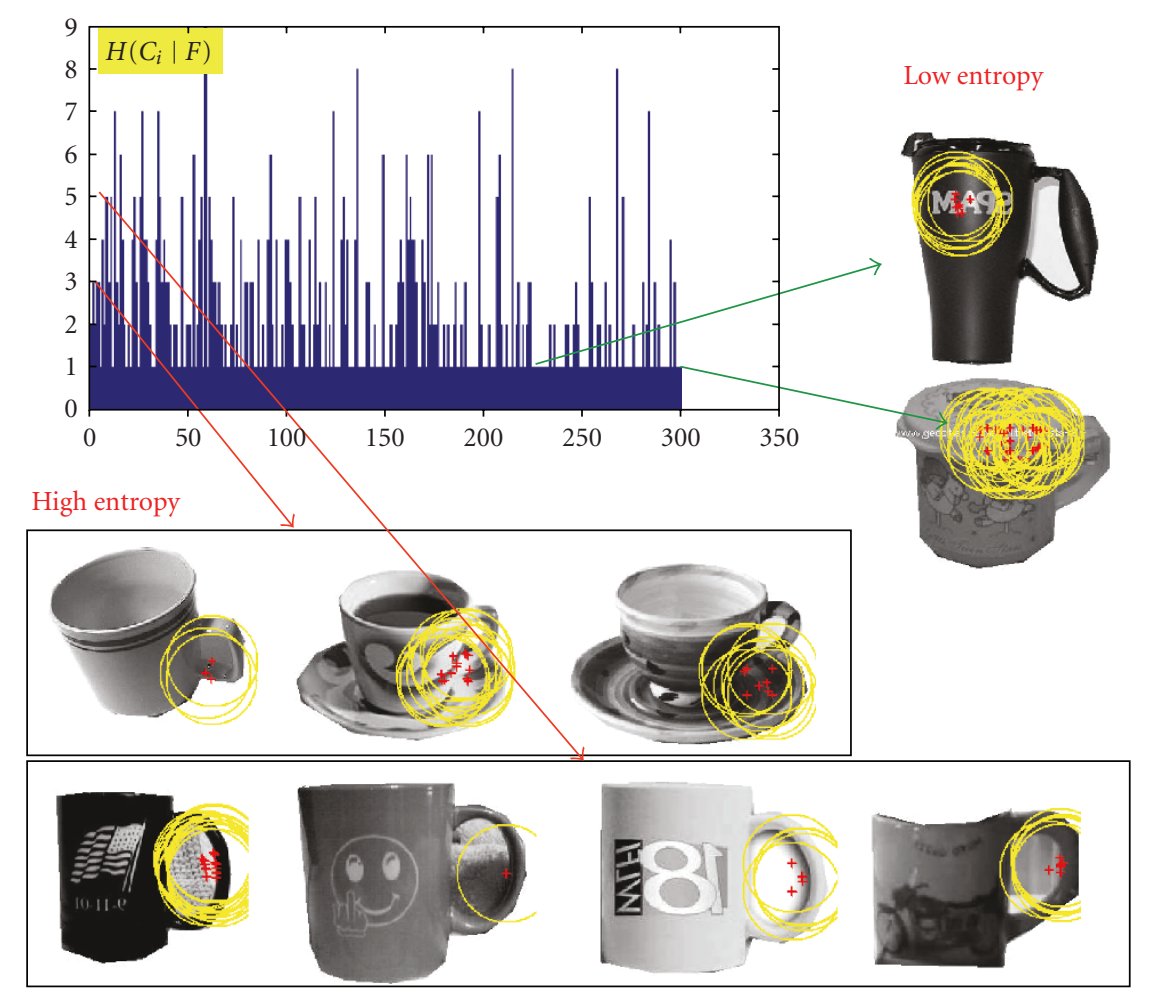

FIGURE 15: Observation for repeatable parts (high entropy) and surface marking parts (low entropy).

4.2. Step 2: Learning Index of CCB Guided by Entropy. We have to learn parameters related to codebook for the likelihood estimation in (4). A codeword in a codebook has four components: codeword index $(F)$, probability of codeword frequency $(\phi)$, appearance parameters (mean, variance for both object and category), and pose parameters (mean, variance for only the object). The codebook selection method is important to achieve successful categorization. We focus on reducing surface markings during visual words or codebook generation, as shown in Figure 13. Our strategies 


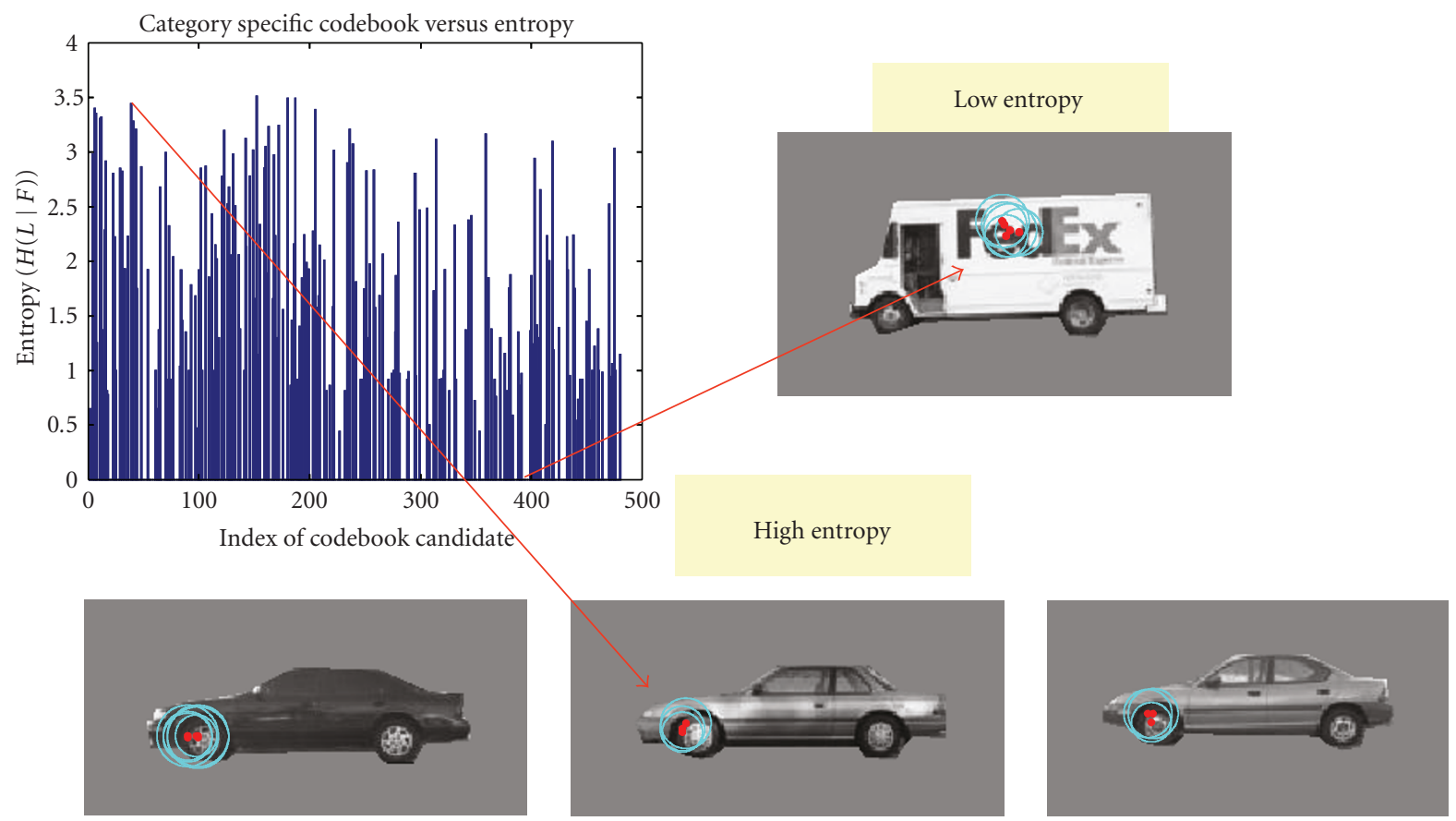

FIGURE 16: Entropy of candidate codebook and corresponding visual features.
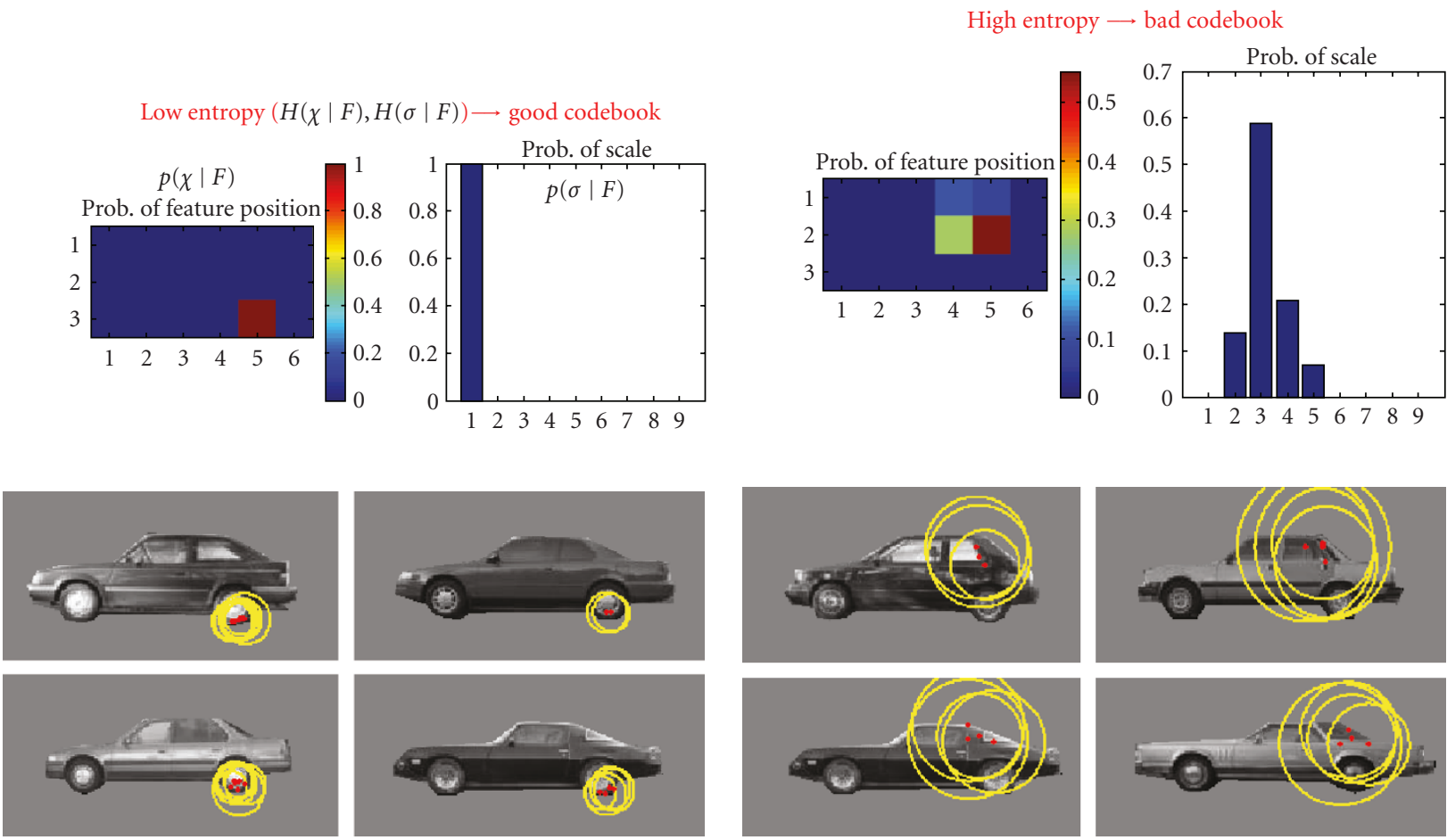

(a)

(b)

Figure 17: Probability distribution of a codeword pose (position and scale) and its corresponding parts. We select the final codebook whose pose entropy is low. 


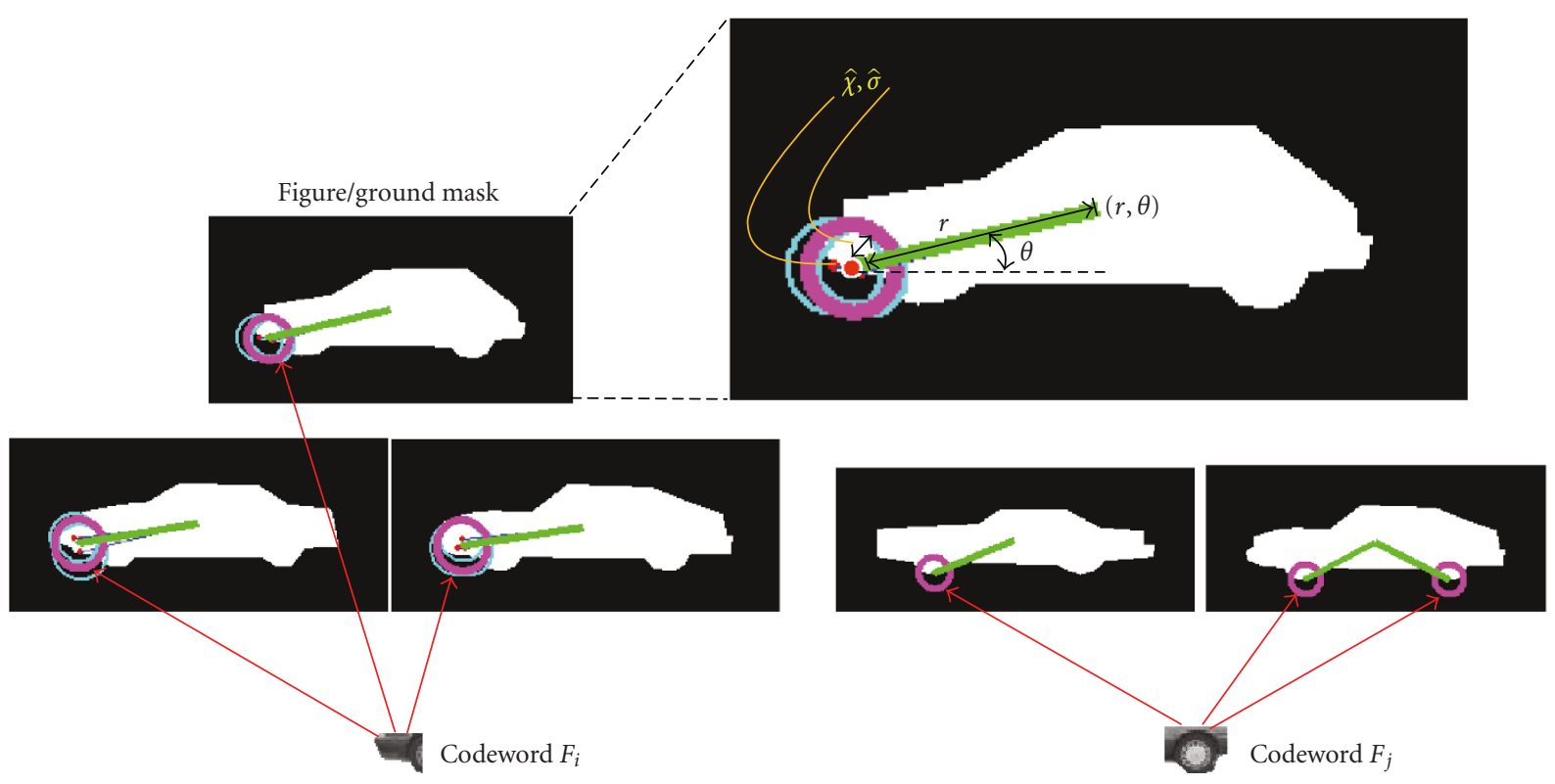

FIGURE 18: Learning CCB pose including figure-ground mask.

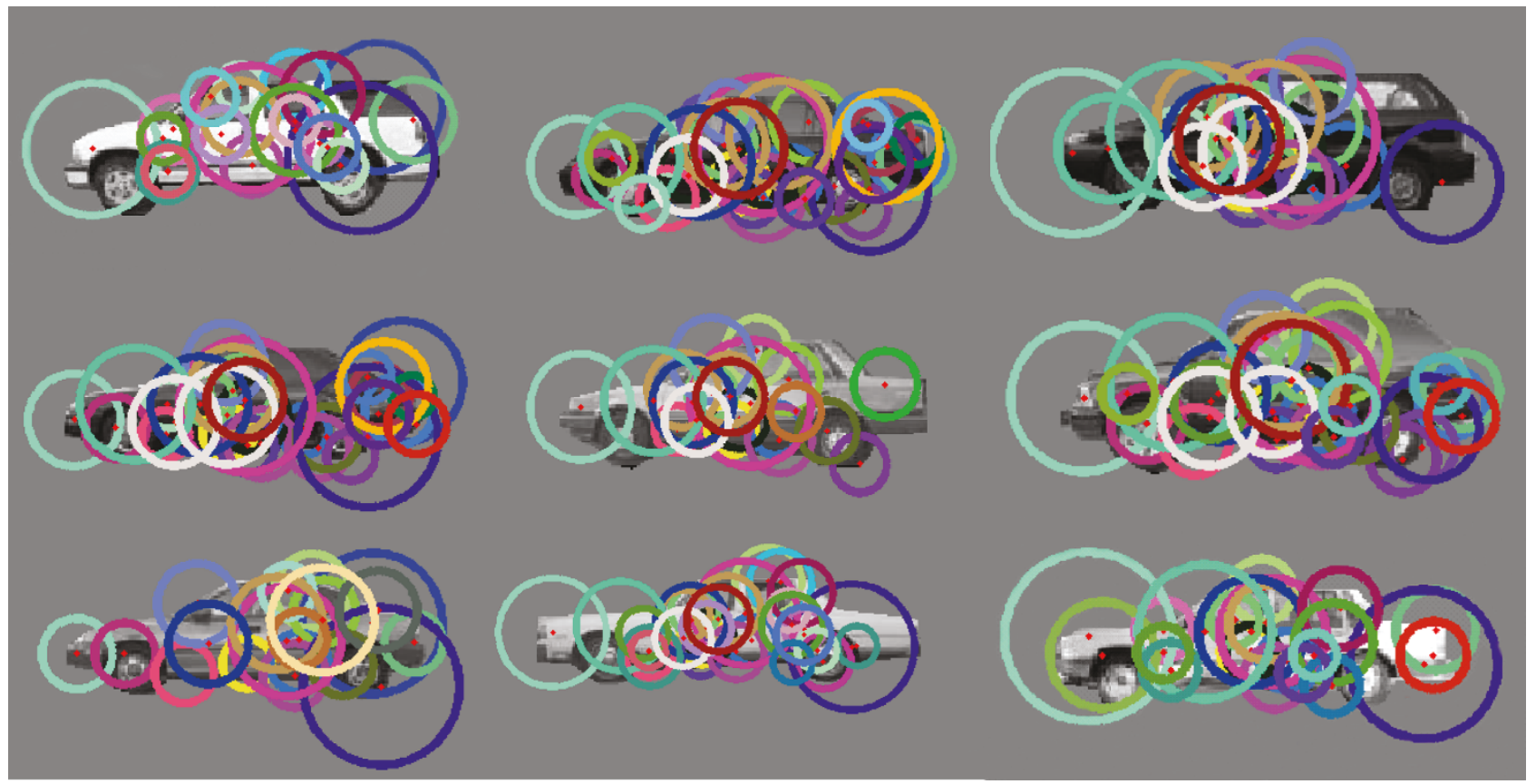

FIGURE 19: Examples of learned codebook overlaid on exemplars. Different color represents different codebook.

are twofold. First, apply intermediate blurring to extract important object shape information. This is motivated from the cognitive experiments showing that human visual systems can categorize blurry objects very quickly and accuracy performance is virtually unaffected by up to $50 \%$ blurring, but then rapidly falls to a low level, following a sharp sigmoid curve $[39,49]$. This means that low spatial frequency information is important to visual categorization. The second is based on the information theory for the codebook selection. The simplest codebook generation method is k-means clustering. However, the proposed entropyguided codebook can represent repeatable or semantically meaningful parts removing surface markings.

In advance, we evaluate the effect of blurring by changing the smoothing level (the standard deviation, $\sigma$ in Gaussian blur). G-RIF features are extracted from the blurred images. Figure 14 shows the evaluation results with the corresponding blurred objects. We use bag-of-keyword method with its nearest neighbor classifier [11]. According to the maximum value, we set the blurring level as $\sigma=3$. 


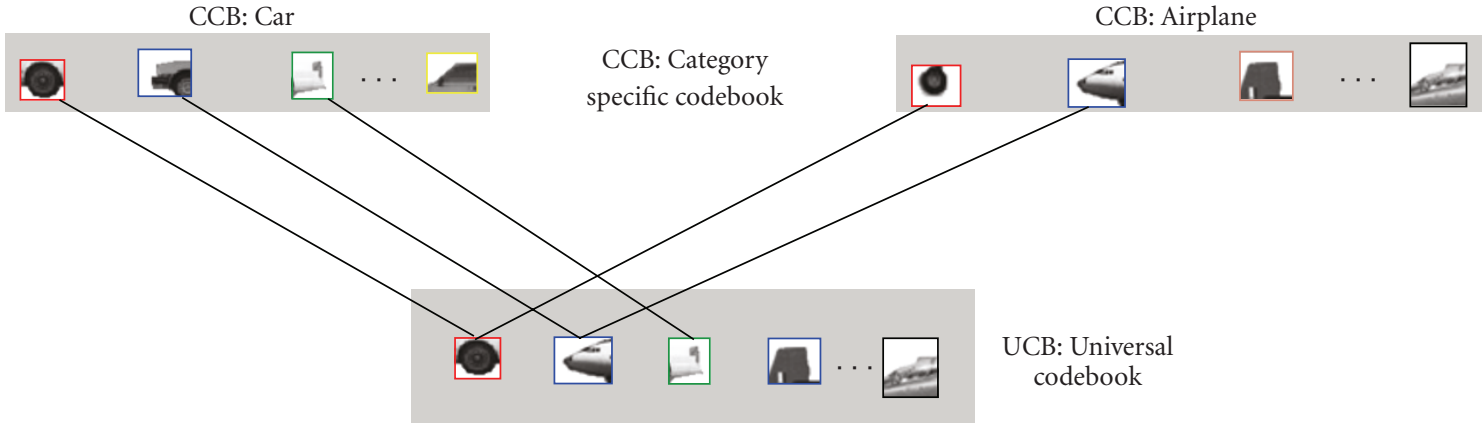

FIGURE 20: Learning universal codebook from category-specific codebooks.

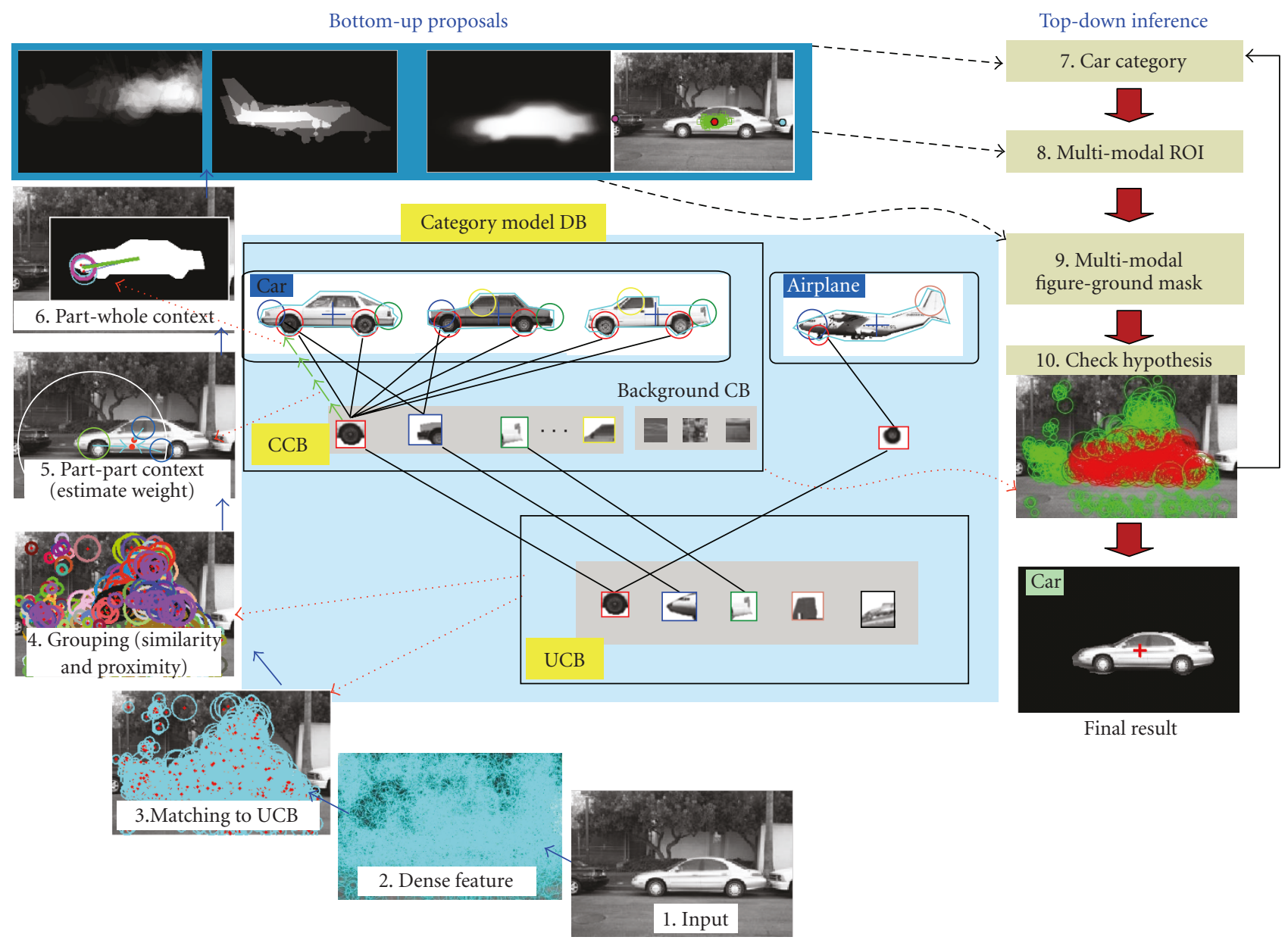

FIGURE 21: The overall inference flow by boosted MCMC method for simultaneous object categorization and figure-ground segmentation.

Assume that we have finite (ex. 15) images. Through agglomerative clustering (bottom-up) and k-means clustering (top-down), we can obtain candidate codebook $F_{\text {hyp }}$. For each codebook candidate $F$, we can estimate entropy of instance label $L$, as (5)

$$
H(L \mid F)=-\sum_{l \in L} p(l \mid F) \log _{2} p(l \mid F),
$$

where $p(l \mid F)$ is the relative frequency of codebook $F$ in object instance $l$.
We have to minimize intraclass variations. As mentioned, one of the main causes of large intraclass variation is surface markings, which have various texture patterns for object instances. Figure 15 represents the relation between entropy of codebook within category and feature positions in category instances for a cup category. Row axis is the ID of codebook and column axis is the entropy value of each codebook within category. As indicated by the arrows, high entropy codebooks are strongly related to semantic, parts and low entropy codebooks are strongly related to 


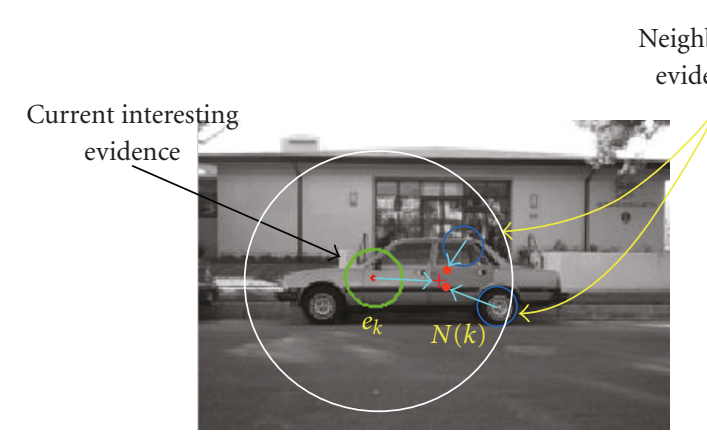

Figure 22: Concept of part-part context. The quality of current interesting evidence is determined by neighboring evidences.

surface markings. So, the surface markings can be removed by finding repeatable parts or high-entropy parts. Figure 16 shows the entropy of the candidate codebook for additional car category. A codeword whose entropy is low belongs to nonrepeatable parts, such as surface markings (see the detected parts in FEDEX) or distinctive parts. A codeword, whose entropy is high, belongs to repeatable (or semantically meaningful) parts, such as the wheel parts.

The candidate codebook is first filtered by entropy values because we also have to consider the statistical property of pose for each codeword. During initial filtering, we select codebook candidates whose entropies are larger than the entropy threshold (0.5, empirically tuned). Based on such a candidate codebook, we check the pose entropy of each codeword. In our object instance representation, the appearance codebook is important to predict the ROI and figure-ground mask. The more stable the part position, the more accurate the estimation obtained. If we quantize part position in the image space and part scale in the scale-space, as shown in Figure 17, we can estimate the probability of part position $p(\chi \mid F)$ for codebook $F$. Likewise, we can estimate the probability of part scale $p(\sigma \mid F)$. Positional entropy and scale entropy are calculated from this probability. Figure 17(a) shows a codeword whose pose entropy (uncertainty) is low and Figure 17(b) shows a codeword whose pose entropy is high. The final codebook is selected by thresholding the pose entropy. We choose the final codebook whose position entropy and scale entropy are less than 1.5 (empirically tuned). The pose entropy is very meaningful to model object categories. If the pose entropy is high for all codebooks, then our joint appearance-shape model is unsuitable, since objects usually have textured (repeated pattern) surfaces. In such a case, the conventional bag of keypoint-based category representation is more suitable, because it discards the spatial distribution of features [11].

4.3. Step 3: Learning Appearance and Pose of CCB. We can obtain a category-specific codebook, including codebook index parameter, through the entropy-guided codebook selection (using appearance entropy and pose entropy). At this state, a finally selected codeword has a set of training features belonging to this codeword. The codebook parameters for appearance are estimated by sample mean $\left(\mu_{a}\right)$ and sample variance $\left(\Lambda_{a}\right)$. For simplicity, we consider only diagonal variance. The parameter estimation of codebook pose is rather difficult, since instances of a codeword can be positioned on different locations in a large image. A Gaussian mixture model can represent such a phenomenon but the complexity of learning increases. We model the codeword pose by compromising a nonparametric and parametric representation scheme, as shown in Figure 18. The sample mean and sample variance of a codeword pose is estimated in polar coordinates from clustered features for each object instance (see the enlarged image). The sample mean is $\mu_{x}=(\hat{\chi}, \hat{\sigma})=((r, \theta), \hat{\sigma}) \cdot r$ denotes the average distance between the considered part and object center of the figure/ground mask. $\theta$ denotes the relative angle of considered part reference on the image row-axis. $\hat{\sigma}$ denotes the estimated standard deviation of pose distribution. This process is repeated for other object instances to which the codeword belongs. We assume a uniform distribution of object instances. Pose information of each codeword is distributed among object instances through such pose estimation process. Figure 19 represents a partial examples of codebook for each instance. Every third codebook is overlaid to discern a different codebook. Colors in the figure represent the ID of codewords. Note that similar parts have the same colors. The parameter estimation $\left(\mu_{a}, \Lambda_{a}\right)$ for the background codebook is almost the same as the foreground codebook, except for the codebook pose. We assume that the pose of the background codebook is randomly distributed in the image space and scale-space.

4.4. Step 4: Learning UCB from CCB. Up until now, we have learned the CCB index, appearance, and pose parameters for each object category. The last learning component is the universal codebook (UCB) index and appearance parameter for bottom-up inference. The learning process is quite simple. As shown in Figure 20, initially we have a set of CCBs, such as a car, or an airplane. The appearance parameter of UCB is estimated by agglomerative clustering used in CCB. Appearance similarity is a useful measure to cluster similar category-specific codewords. In Figure 20, a front wheel of a car category and a wheel of an airplane have similar appearance. Therefore, appearance of two category-specific codewords merges into a universal codeword. Following this process, each universal codeword has the link information between itself and indices of category-specific codewords. The link information is useful during bottom-up inference, as explained in the next section.

4.5. Prior for Category, ROI, and Mask. Prior distributions in (2) are learned using a set of labeled training images. Let trained database $D$ have category label $C_{D B}$, ROI $V_{D B}$, and figure-ground mask $M_{D B}$ for each instance. At this state, parameters related to codebook $(\phi, \mu, \Lambda)$ are null. If there are $N_{C}$ categories and each category has $N_{M}$ examples, then the category prior $p(C \mid D)$ is uniform as $1 / N_{C}$. Given a category, the viewpoint distribution can be estimated directly from labeled examples. However, we define $p(V \mid C, D)$, as $p\left(x_{c}, y_{c}, s \mid C, D\right)=1 / A \cdot 1 / 1.5$ for the generalization. 


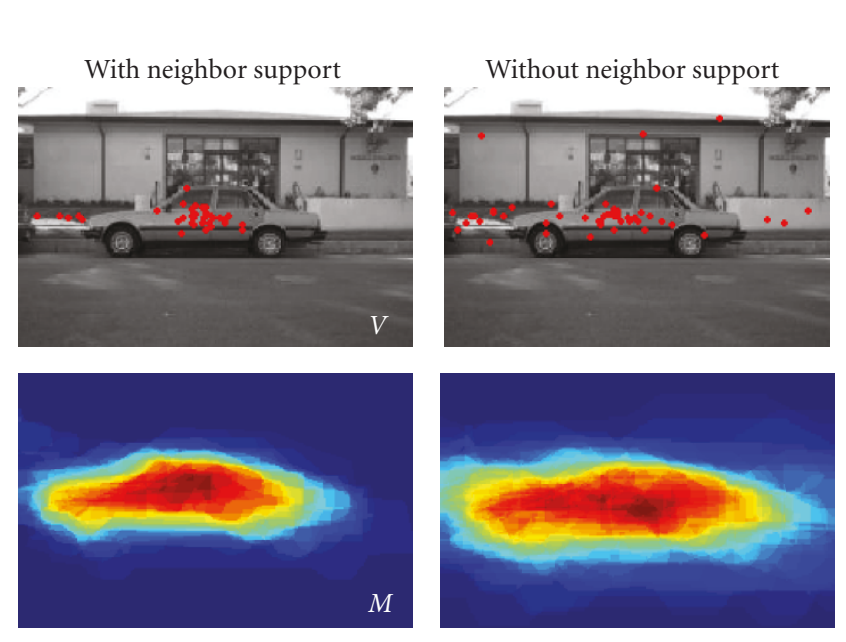

(a) The effect of part-part context
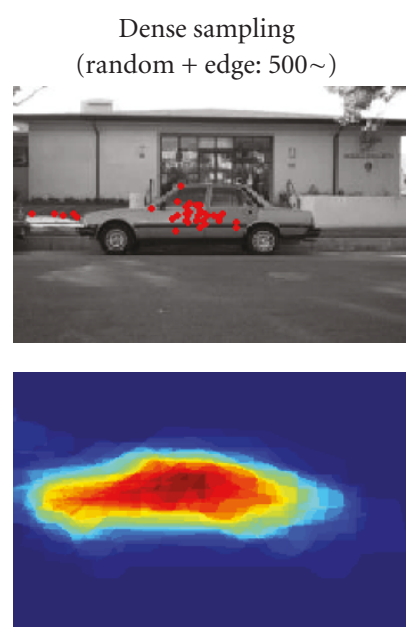

(b) The effect of feature point sampling
Sparse sampling (DoG + Harris: about 100)
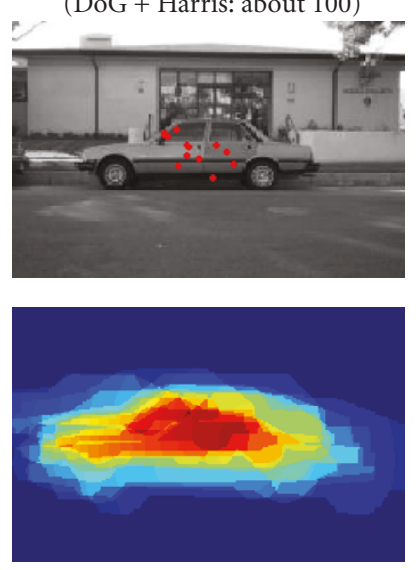

FIGURE 23: Properties of online boosting methods: (top row) estimated ROI (object center) points, (bottom row) accumulated figure/ground masks.

$A$ represents the area of search region. In a real environment, objects can be anywhere in an image. We restrict the scale factor in the range of [0.5 2]. Given category label, viewpoint, and figure-ground masks in $D$, the prior $p(M \mid C, V, D)$ is defined as $1 / N_{M}$, since we randomly choose the figureground mask in the database.

\section{Statistical Inference by Boosted MCMC}

We can obtain optimal object categorization and figureground segmentation by solving (1). However, due to the high dimensionality, direct inference is intractable. We utilize the approximate inference method using a sampling method, such as Markov Chain Monte Carlo (MCMC) [50]. MCMC samples guarantee convergence to the posterior distribution. The Metropolis-Hastings (M-H) algorithm is often used for MCMC inference. The original MCMC can provide a globally optimal solution with the cost of a long time (many samples). We utilize M-H sampling but we modify the proposal function $\left(q\left(H \rightarrow H^{\prime}\right)\right)$ by multimodal distribution. It consists of prior distribution and boosted distribution from bottom-up inference (see the dotted arrows in Figure 11(b)). Samples from multimodal distribution are accepted with probability $\alpha$, defined as (6). Figure 21 shows the overall inference flow graphically. Details of the bottom-up proposal and multimodal sampling-based inference are explained in the following subsections.

$$
\alpha=\min \left\{1, \frac{p\left(H^{\prime} \mid G, D\right)}{p(H \mid G, D)} \cdot \frac{q\left(H^{\prime} \rightarrow H \mid G, D\right)}{q\left(H \rightarrow H^{\prime} \mid G, D\right)}\right\} .
$$

\subsection{Bottom-Up Proposal by Context-Based Boosting}

5.1.1. Dense Feature Grouping Using Similarity and Proximity. First, we extract local features at dense points, such as corner, blob center, and edge samples as shown in Figure 21 ((2) Dense feature). The average number of features per $320 \times 240$ image is 1000. It is inefficient to directly use such a huge number of features for bottom-up inference. Instead, we filter out the dense features using discrimination by the $\mathrm{k}$ $\mathrm{NN}$ (nearest neighbor, in this paper $k=1$ ) classifier with UCB ((3) Matching to UCB). Then filtered dense features are grouped according to Gestalt's law of appearance similarity and proximity ((4) Grouping: similarity and proximity). Similar features within 25 pixels are grouped. We denote the finally grouped features as e. In Figure 21, the image denoted as (4) Grouping shows the clustered features with the color index of UCB.

5.1.2. Online Boost Using Visual Context. Given evidence (e, clustered from dense features), we can directly estimate the proposal function bottom-up using two kinds of visual context. The first context is part-whole relation, which is a sort of hierarchical context. Evidence, $e_{k}$, can predict a codeword in UCB. Since UCB contains CCB links, we can predict category $(C)$, ROI $(V)$, and figure-ground mask $(M)$. Figures 20 and 18 will help you understand the partwhole prediction mechanism. The second context is the partpart relation. As shown in Figure 22, the quality of current interesting evidence, $e_{k}$, is affected by neighboring evidences $N(k)$. We can predict ROI of $e_{k}$ using the part-whole context. Neighboring evidences can also provide ROI (object center, relative scale). If these ROIs are compatible to the ROI by $e_{k}$, then we accept the prediction of the current evidence. Based on the concept of visual contexts, we can model this phenomenon mathematically by borrowing the concept of boosting [51]. In the original boosting, a strong classifier $(g)$ is constructed from a set of weak classifiers $\left(h_{k}\right)$, as $g(x)=$ $\sum_{k=1}^{k_{\max }} \alpha_{k} h_{k}(x)$. The weak classifier weight $\alpha_{k}$ is learned off line using a positive and negative training set.

The joint category and ROI classifier $g(C, V, M \mid \mathbf{e})$ is defined in (7). Given an input evidence $e_{k}$, we can predict category $(C)$, ROI $(V)$, and figure-ground mask $(M)$ using the part-whole context, such as evidence to UCB, UCB to 

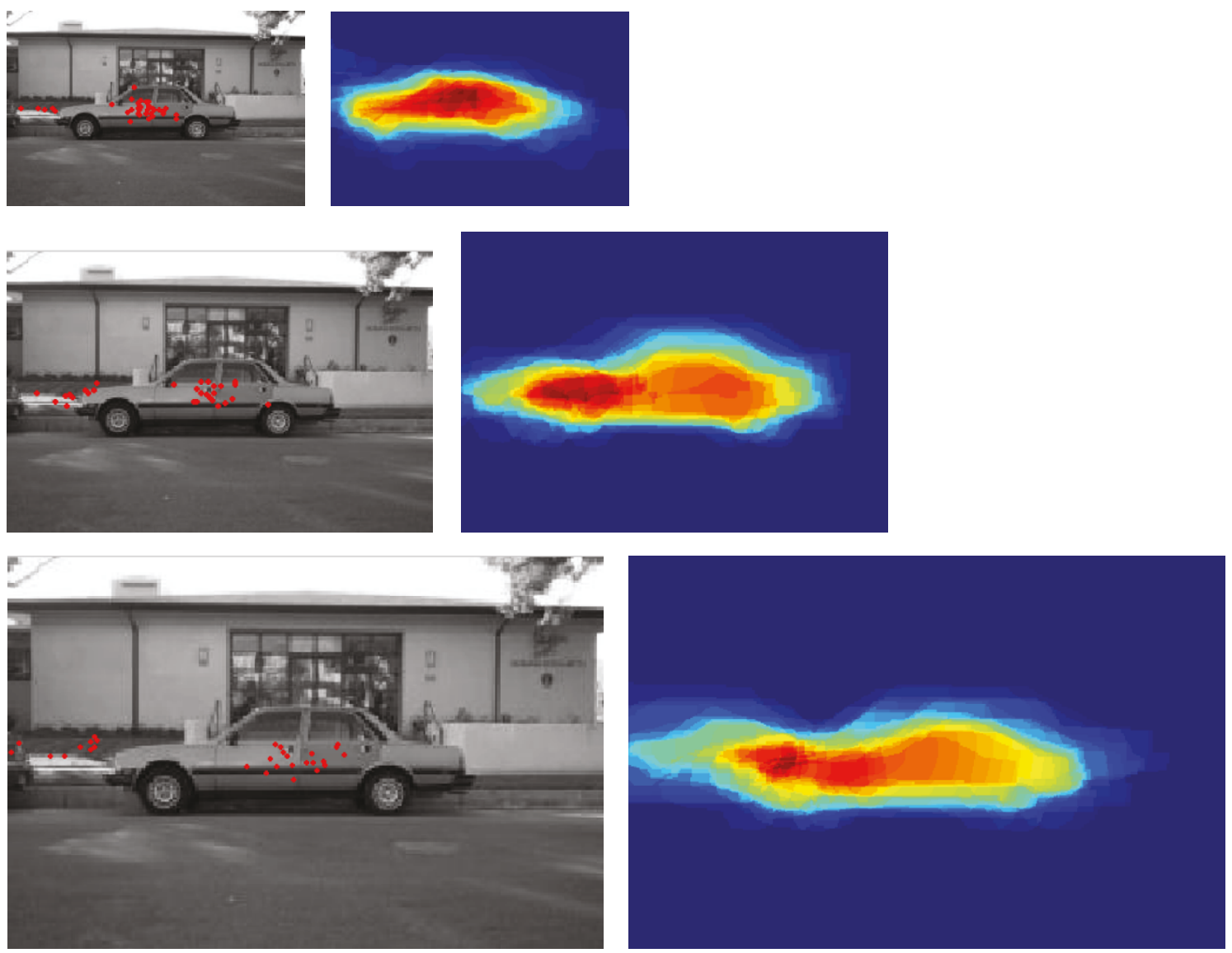

FIGURE 24: Robustness for scale changed test set: (first column) estimated ROI points, and (second column) accumulated figure/ground masks.

CCB, and CCB to the object instance in DB. $L_{i}$ denotes all possible interpretation links. We assume $p(C, V, M$ । $\left.I_{i}\right), p\left(I_{i} \mid e_{k}\right)$ to be uniform for simplicity. The part-part context is utilized to estimate the weight $\alpha_{k}$ of the weak classifier (parenthesis in (7)). Compared to the conventional off-line learning $\alpha$, this is learned online, using neighboring evidences. Thus we term our bottom-up inference, online boost. The $\alpha_{k}$ for the weak classifier is defined as $\alpha_{k}=$ $n_{\text {support }} /|N(k)|$, where $n_{\text {support }}$ is the support count from evidences $N(k)$.

$$
g(C, V, M \mathbf{e})=\sum_{k=1}^{k_{\max }} \alpha_{k}\left(\sum_{i} p\left(C, V, M L_{i}\right) p\left(L_{i} e_{k}\right)\right)
$$

We increase the support count if $\mid$ center $(k)-\operatorname{center}(j) \mid<$ $\delta$, where $j \in N(k)$. center $(k)$ represents a predicted object center position using $e_{k}$, and center $(j)$ represents a predicted object center position using $e_{j}$ in $N(k)$. Empirically, we can obtain good estimation if we quantize the $\alpha_{k}$. We set $\hat{\alpha}_{k}=1, \alpha>0.5$; otherwise, $\hat{\alpha}_{k}=0$. This can remove outliers robustly. Figure 23(a) shows the effect of part-part context in bottom-up boosting. Note the role of part-part context in online boosting of category, ROI, and figureground mask. Such online boosting is quite similar to voting in $(C, V, M)$ space. With this bottom-up inference method, we also compare sampling methods of feature points: dense sampling (Harris + DoG points + random + edge samples) and sparse sampling (Harris + DoG points only) in scale space. Figure 23(b) shows an example of bottom-up boosting with two kinds of sampling. Dense sampling-based boosting shows more stable evidence. Figure 24 shows the robustness to scale changes in bottom-up boosting. In this small test set, we can conclude that our part-part context, dense sampling in scale-space is important to achieve stable bottom-up inference.

5.1.3. Estimation of Bottom-Up Proposal Function. Given voting results of $g(C, V, M)$, we can estimate the bottom-up proposal function that is used in MCMC optimization. We need three conditional proposal distributions as indicated in Figure 11(b) (dotted arrows). The bottom-up proposal $\left(q_{\text {boost }}(C \mid \mathbf{e})\right)$ for object category is the relative count of evidence votes as $(8)$.

$$
q_{\text {boost }}(C \mid \mathbf{e})=\frac{\text { No. of votes to } C}{\text { Total No. of votes }} .
$$

Given category label $C$, the ROI distribution $\left(q_{\text {boost }}(V \mid\right.$ $C, \mathbf{e})$ ) is estimated directly from mean-shift clustering for 

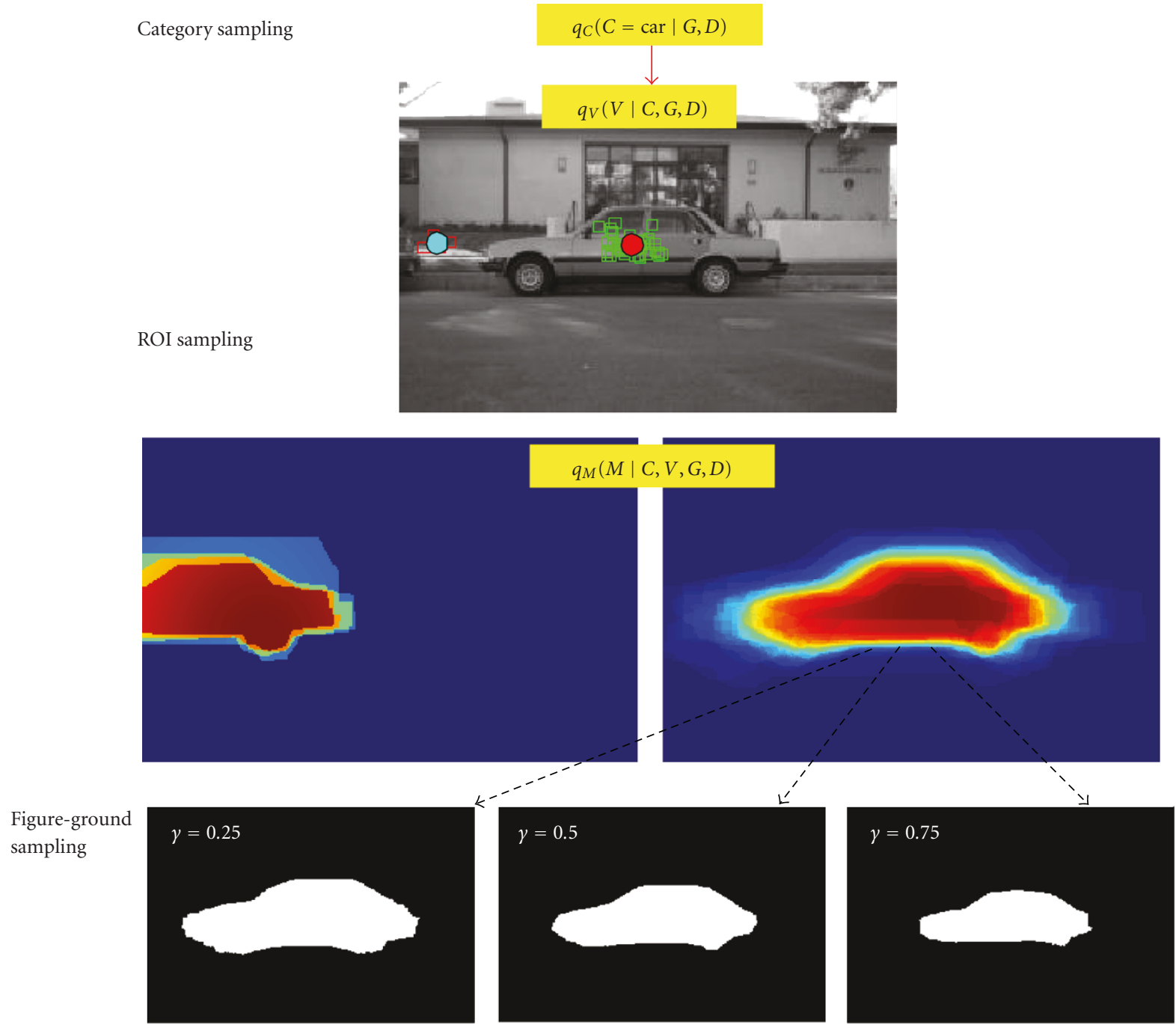

FIGURE 25: Examples of proposed distribution: category sampling, ROI sampling, and figure-ground sampling.

a set of viewpoints belonging to category $C[52] . \mathcal{N}$ denotes the Gaussian distribution.

$$
\begin{aligned}
q_{\text {boost }}(V(\chi, s) \mid C, \mathbf{e})= & \sum_{m} \pi_{m} \mathcal{N}_{\chi, m}\left(\chi ; \mu_{\chi}, \sigma_{\chi}^{2}\right) \\
& \cdot \mathcal{N}_{\mathrm{s}, m}\left(s ; \mu_{s}, \sigma_{s}^{2}\right) .
\end{aligned}
$$

Finally, given object category and ROI, we assume that the proposal distribution of the figure-ground mask is uniform, as $q_{\text {boost }}(M \mid C, V, \mathbf{e})=1$. An instance of mask $M$ is obtained by randomly thresholding $(\gamma)$, the voting values of figure-ground masks. The voting values are normalized by the maximal vote, so $\gamma$ is in the range of [ $\left[\begin{array}{ll}0 & 1\end{array}\right]$.

The proposed online boosting for MCMC proposals is quite similar to other voting-based approaches. In general, a voting method provides a vote if a similarity is smaller than a predefined threshold. The proposed online boosting is similar at this point. However, we give a weight to the voting value based on the spatial contexts such as part-whole and part-part contexts.
5.2. Top-Down Inference by Multimodal MCMC. The performance of MCMC-based inference depends on the sampling method. In this section, we propose a multimodal MCMCsampling method for fast and accurate inference. The multimodal proposal functions are defined as (10), using prior distributions learned from training data and boosted proposal distributions in $(8),(9) . \beta_{i}$ is the mixing probability for each random variable sampling. We usually set them as 0.5 .

$$
\begin{aligned}
q\left(H \longrightarrow H^{\prime} \mid G, D\right)= & q_{C}(C \mid G, D) q_{V}(V \mid C, G, D) \\
& \times q_{M}(M \mid C, V, G, D), \\
q_{C}(C \mid G, D)= & \beta_{1} p(C \mid D)+\left(1-\beta_{1}\right) q_{\text {boost }}(C \mid G, D), \\
q_{V}(V \mid C, G, D)= & \beta_{2} p(V \mid C, D) \\
& +\left(1-\beta_{2}\right) q_{\text {boost }}(V \mid C, G, D),
\end{aligned}
$$




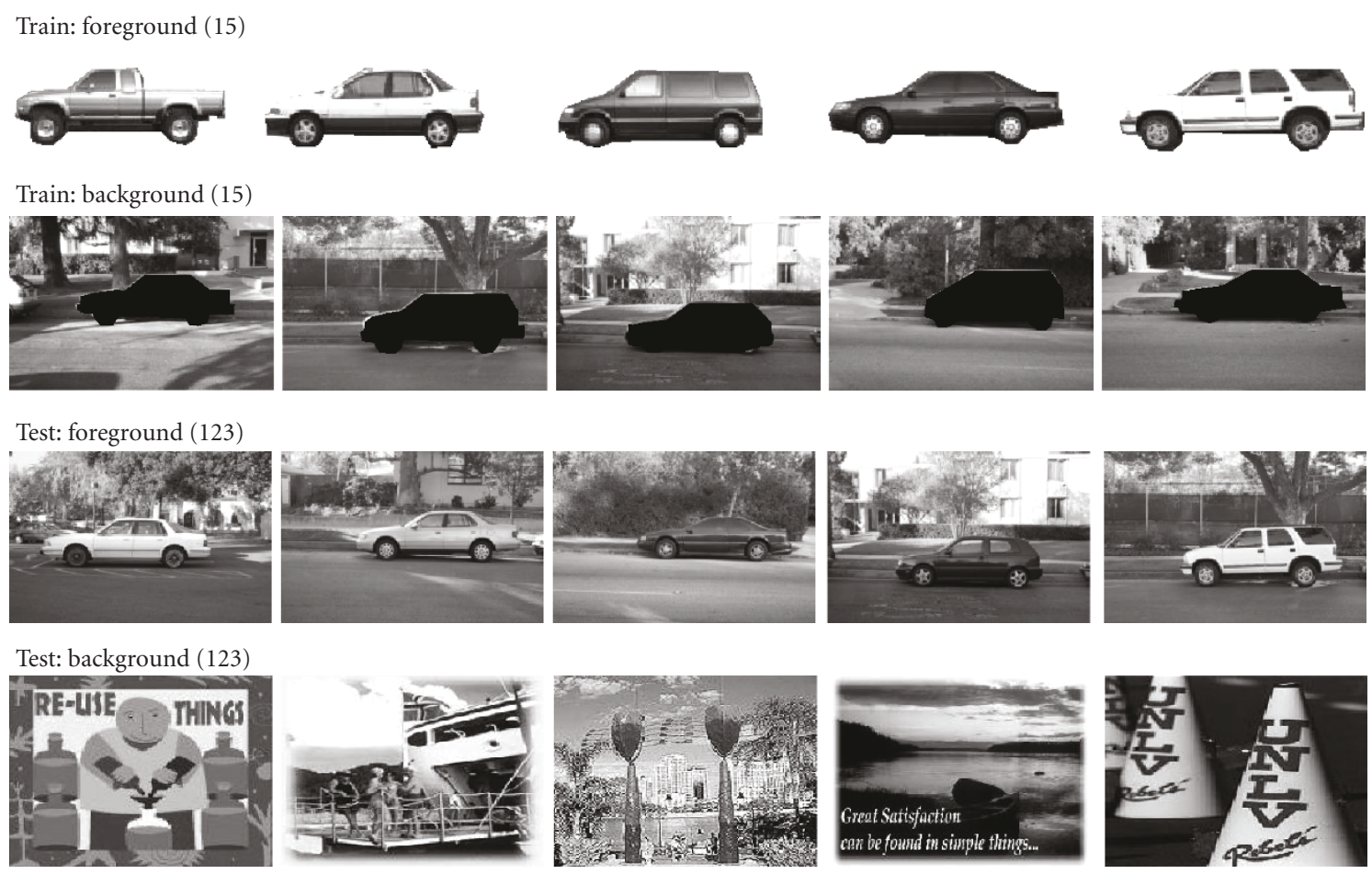

Figure 26: Partial examples of training set and test set for car category.

$$
\begin{aligned}
q_{M}(M \mid C, V, G, D)= & \beta_{3} p(M \mid C, V, D) \\
& +\left(1-\beta_{3}\right) q_{\text {boost }}(M \mid C, V, G, D) .
\end{aligned}
$$

We can generate a hypothesis $H^{\prime}$, as shown in Figure 25, through conditional sampling from multimodal distributions. Then, we can calculate the likelihood using (3), (4). Figure 21 (right figure) shows figural features (red color) and background features (green color) divided by hypothesis $H^{\prime}$. The hypothesis $\left(H^{\prime}\right)$ is accepted with probability $\alpha$ in (6). After convergence, we can obtain optimal inference result by expectation of accepted samples.

\section{Experimental Results}

In the first experiment, we compare two inference methods for simultaneous object categorization and segmentation: bottom-up only and bottom-up + top-down. We use the ROC (receiver operating characteristic) curve as a performance measure [53]. We use the Caltech Car side dataset for the evaluation (http://www.vision.caltech .edu/Image_Datasets/Caltech101/Caltech101.html). 15 randomly selected foreground and background images are used to learn our inference system. In the background image, we extract features only of background regions. We test 123 cluttered car images as the foreground and 123 Google images as the background, as shown in Figure 26. It is important to define the control threshold for the correct ROC curve generation. Since our research goal is to categorize and figure-ground segmentation simultaneously,
TABLE 1: Summary of EER performance for car category detection.

\begin{tabular}{lccc}
\hline & Ours & {$[54]$} & {$[5]$} \\
\hline car side & $89.0 \%$ & $87.3 \%$ & $88.0 \%$ \\
EER criteria & Label + region & label only & label only \\
\hline
\end{tabular}

we use one control parameter and two thresholds. Meanshift clustering (window radius 30) can provide clustered ROI (object center points). We use this number $(k)$ as the main control parameter. We define an inference as being a correct positive if $k>k_{\mathrm{th}}$, the ROI center error is less than 50 pixels, and the region overlap error $\left(1-\left(R_{E} \cap R_{T}\right) /\left(R_{E} \cup R_{T}\right)\right)$ is less than $30 \%$, where $R_{E}$ is the region of estimation and $R_{T}$ is the ground truth region. In bottom-up with top-down method, we use the same control parameter with additional likelihood ratio test $p(G \mid O) / P(G \mid B)$, where $G$ denotes input features, $O$ denotes object hypothesis, and $B$ denotes background hypothesis.

We apply 123 images for the positives set and 123 images for the negative set based on such settings. By controlling the threshold $k_{\text {th }}$ from 0 to 100 , we can obtain ROC curve, like Figure 27(a). The equal error rate (EER) for bottom-up only is $73 \%$ and that for bottom-up with the top-down method is $89 \%$. At this EER, $k_{\mathrm{th}}$ is 8 . Table 1 summarizes EER results compared to other related methods. Our EER is higher than that of the others. Furthermore, our system can categorize and segment figure-ground. Figure 27(b) shows the partial car detection results.

As a next evaluation, we check the detection performance under object occlusion. For this test, we randomly select 50 


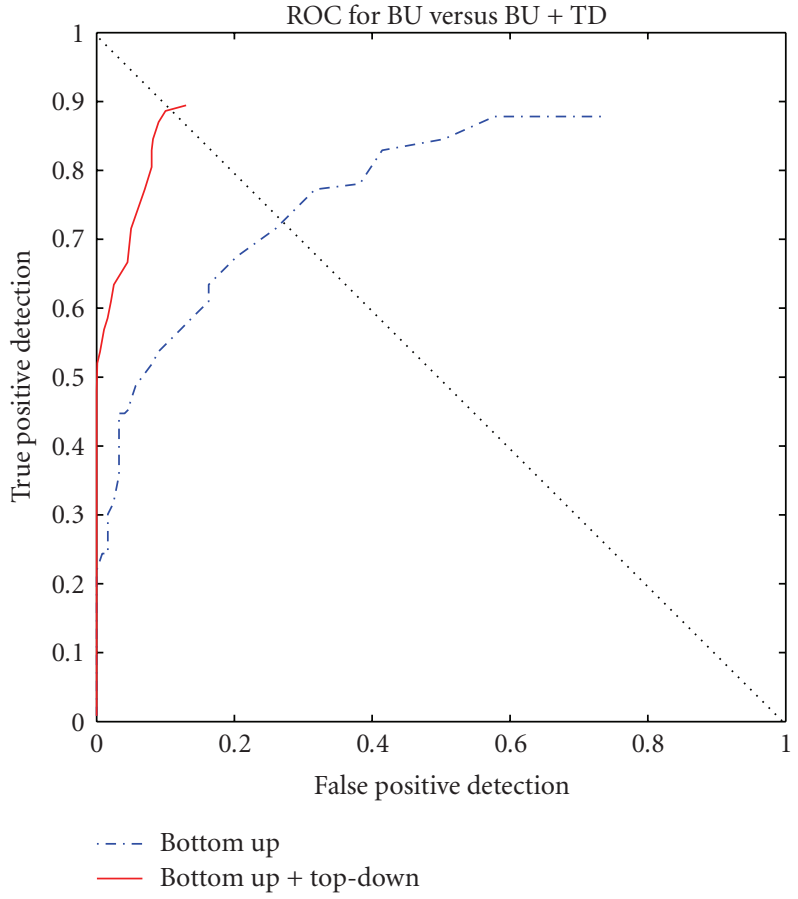

(a) ROC curve for car category
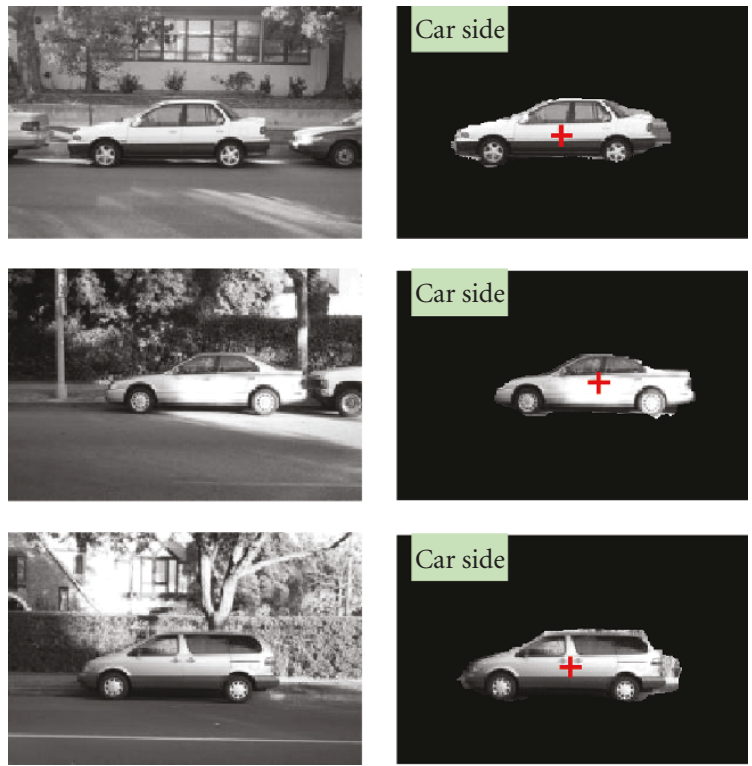

(b) Detection results

FIGURE 27: ROC curve for car detection and test results.

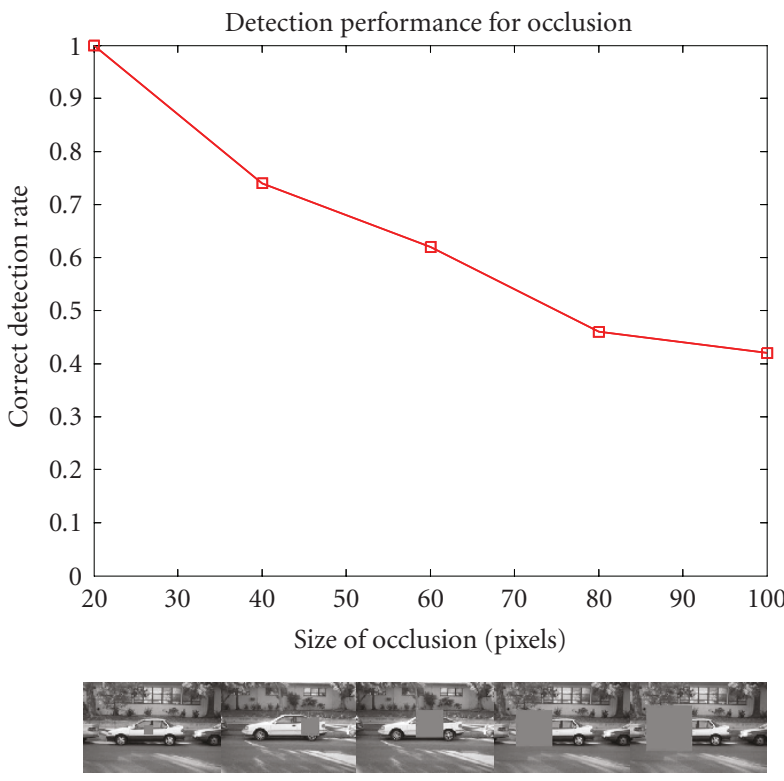

(a) Performance for car occlusion
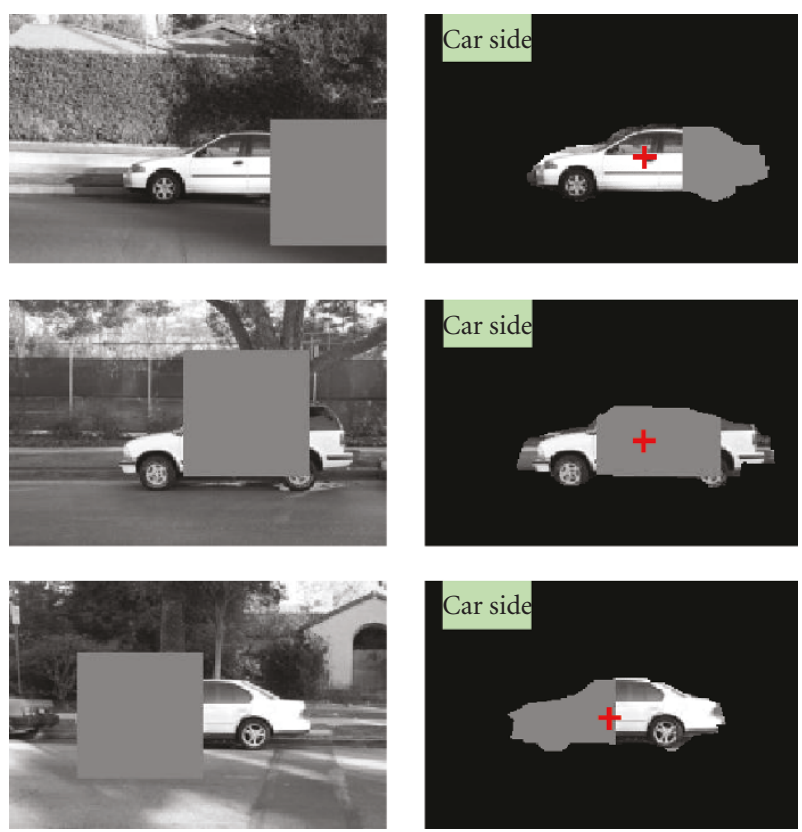

(b) Detection results

FIGURE 28: Detection performance under occlusion and several detection results.

test images and add artificial squares sized from 20 to 100 pixels in random positions. The average car length is 170 pixels. We use the parameters selected at EER. Figure 28(a) represents the evaluation results. Note that our system is relatively robust to occlusion. Figure 28 (b) shows successfully detected and segmented results of the car category. Our system can predict the shape for the occluded regions (see the bottom in Figure 28(b)).

We also evaluate our system for the Caltech face data set (http://www.robots.ox.ac.uk/vgg/data3.html). The face DB 

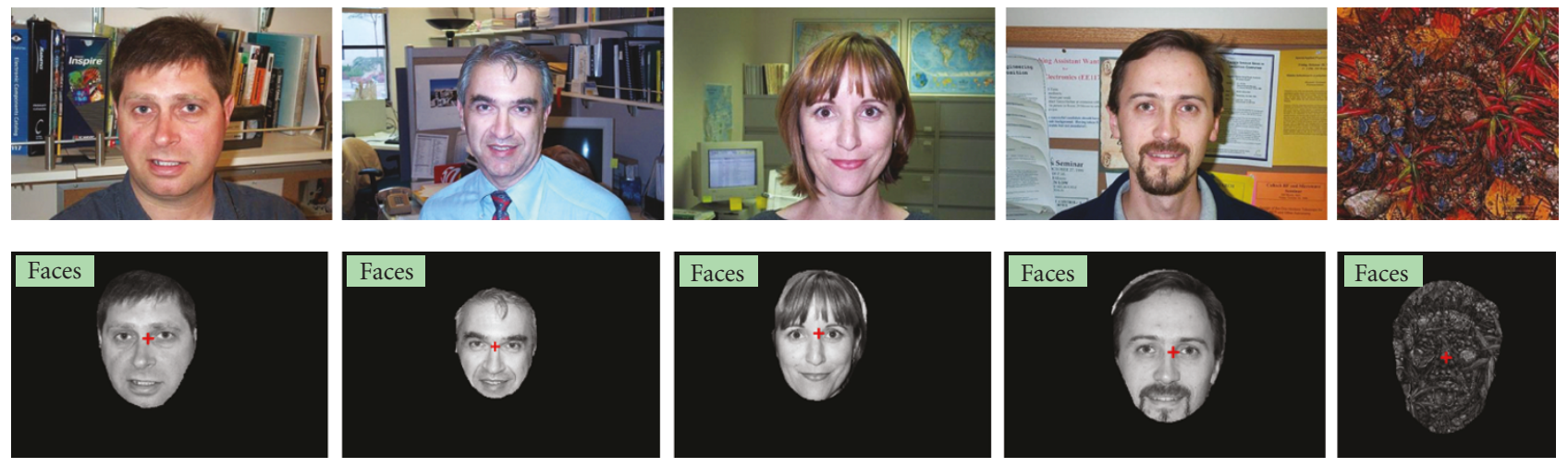

Figure 29: Examples of face detection and segmentation.

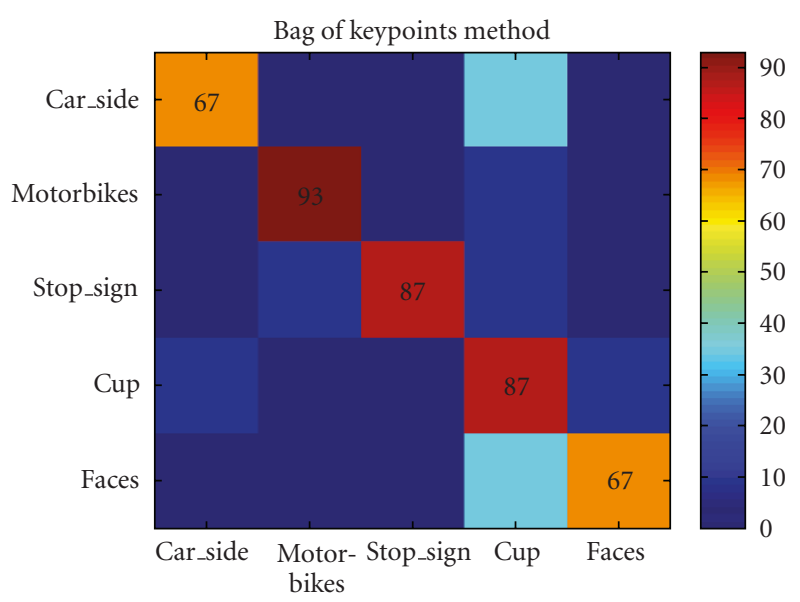

(a) Bag of features: $80 \%$

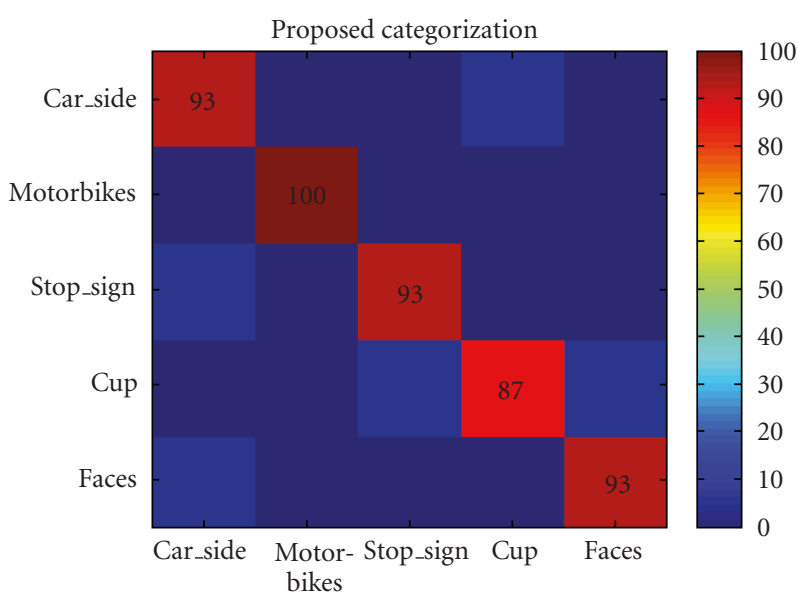

(b) Proposed method: $93.3 \%$

Figure 30: The improvement of categorization.

consists of 435 faces with clutter and 468 background images. Training is conducted using only 15 random selections. 200 novel face images and 200 novel background images are used to check EER. We use the parameters selected in EER for car detection. Table 2 summarizes the training set composition and EER performance. Unsupervised learning requires a very large amount of training data to provide comparable performance of ours $[5,55]$. A partially segmented set can reduce the amount of unsegmented training data [30]. Our system relies on a fully segmented small training set (just 15 images) that provides better performance. Figure 29 shows partial examples of face categorization and figure-ground segmentation results. Through this experiment, we found that our system can detect faces robustly for various facial expressions and backgrounds. The last example is quite interesting. Our algorithm can detect human faces from cluttered images, just as human vision can!

In addition, we evaluate our system in terms of categorization performance for selected five Caltech categories (car, motorbike, stop sign, cup, and faces). In this experiment, we use 15 randomly selected images (segmented) for training and test 15 randomly selected unlearned images. Figure 30 shows confusion matrices using the bag of features and
TABLE 2: Composition of training set and EER for face test set.

\begin{tabular}{lccc}
\hline Method & no. train (unseg) & no. train (seg) & EER \\
\hline$[55]$ & 200 & 0 & $94.0 \%$ \\
{$[5]$} & 220 & 0 & $96.4 \%$ \\
{$[30]$} & 50 & 10 & $96.5 \%$ \\
Ours & 0 & 15 & $97.3 \%$ \\
\hline
\end{tabular}

ours. Note that our method perform better with additional figure/ground information. Figure 31 shows categorization and segmentation results for real world images using trained parameters with the Caltech DB.

\section{Conclusion}

In this paper, we proposed an integrated method for object categorization and figure-ground segmentation for unknown novel objects motivated from human visual systems, especially visual contexts. Simultaneous categorization and segmentation is difficult under large intraclass variation and background clutter. We solve such issues by 

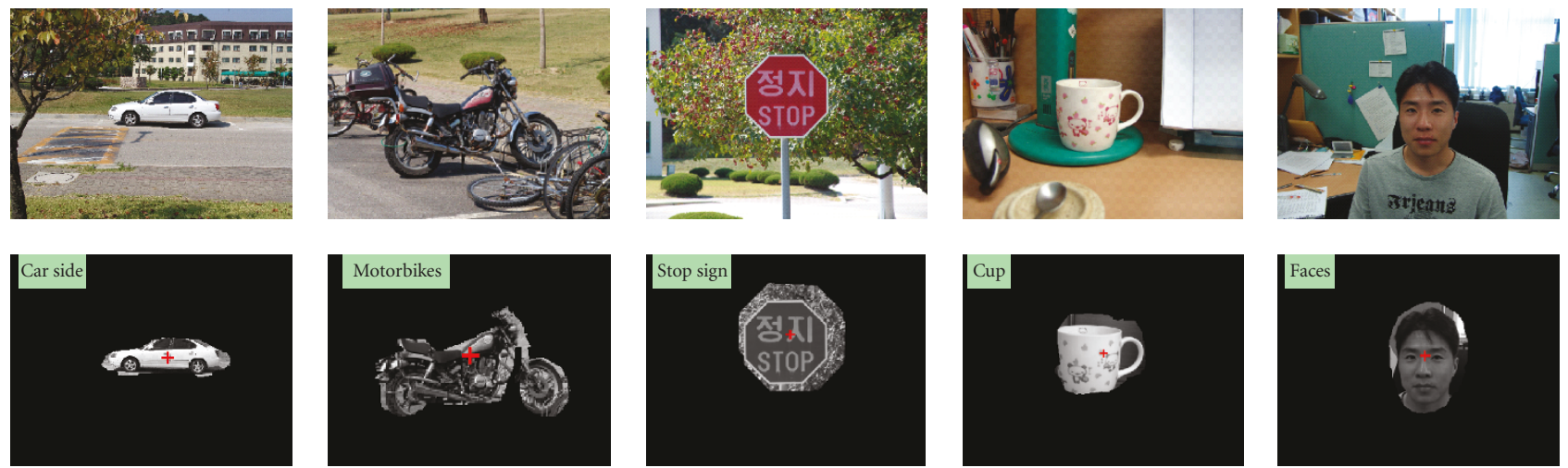

FIGURE 31: Categorization and segmentation results for real-world images.

utilizing part-part context, part-whole context, and objectbackground context to reduce the effect of background clutter. Part-part context can remove or reduce the effect of outliers, and part-whole context can predict the category label and region of interest with the figure-ground mask. By accumulating weak classifiers, we can boost the bottom-up inference. For top-down inference, we propose a multimodal MCMC sampling method. Samples are selected from a multimodal distribution composed of a prior term and a bottom-up proposal term. This method converges to an almost global solution. Through various evaluations, we conclude that our integrated system is useful in the object categorization and figure-ground segmentation issue. We are currently pursuing how to relate object identification and categorization based on our object categorization results. Object categorization obtains similarity information from object instances. Likewise, object identification can update its object instances from object categorization results developed in this work. If we research the cooperative relationship further, both research areas will have synergetic effects.

\section{Acknowledgment}

This research was supported by Yeungnam University research grants in 210-A-054-014.

\section{References}

[1] Z. Lin, S. Kim, and I. S. Kweon, "Recognition-based indoor topological navigation using robust invariant features," in Proceedings of IEEE/RSJ International Conference on Intelligent Robots and Systems (IROS '05), 2005.

[2] J. Košecká and F. Li, "Vision based topological Markov localization," in Proceedings of the IEEE International Conference on Robotics and Automation (ICRA '04), vol. 2, pp. 1481-1486, April-May 2004.

[3] D. G. Lowe, "Distinctive image features from scale-invariant keypoints," International Journal of Computer Vision, vol. 60, no. 2, pp. 91-110, 2004.

[4] S. Kim, K. J. Yoon, and I. S. Kweon, "Object recognition using a generalized robust invariant feature and Gestalt's law of proximity and similarity," Pattern Recognition, vol. 41, no. 2, pp. 726-741, 2008.
[5] R. Fergus, P. Perona, and A. Zisserman, "Object class recognition by unsupervised scale-invariant learning," in Proceedings of the Computer Society Conference on Computer Vision and Pattern Recognition (CVPR '03), vol. 2, pp. 264-271, Madison, Wis, USA, June 2003.

[6] K. Mikolajczyk, B. Leibe, and B. Schiele, "Multiple object class detection with a generative model," in Proceedings of the IEEE Computer Society Conference on Computer Vision and Pattern Recognition (CVPR '06), vol. 1, pp. 26-33, New York, NY, USA, June 2006.

[7] J. Zhang, M. Marszałek, S. Lazebnik, and C. Schmid, "Local features and kernels for classification of texture and object categories: a comprehensive study," International Journal of Computer Vision, vol. 73, no. 2, pp. 213-238, 2007.

[8] B. Leibe and B. Schiele, "Analyzing appearance and contour based methods for object categorization," in Proceedings of the IEEE Computer Society Conference on Computer Vision and Pattern Recognition (CVPR '03), vol. 2, pp. 409-415, Madison, Wis, USA, June 2003.

[9] P. Moreels, M. Maire, and P. Perona, "Recognition by probabilistic hypothesis construction," in Proceedings of the European Conference on Computer Vision, vol. 3021 of Lecture Notes in Computer Science, pp. 55-68, 2004.

[10] A. C. Berg, T. L. Berg, and J. Malik, "Shape matching and object recognition using low distortion correspondences," in Proceedings of the Computer Society Conference on Computer Vision and Pattern Recognition (CVPR '05), vol. 1, pp. 26-33, San Diego, Calif, USA, June 2005.

[11] G. Csurka, C. R. Dance, L. Fan, J. Willamowski, and C. Bray, "Visual categorization with bags of keypoints," in Proceedings of the Workshop on Statistical Learning in Computer Vision (ECCV'04), 2004.

[12] J. Winn, A. Criminisi, and T. Minka, "Object categorization by learned universal visual dictionary," in Proceedings of the 10th IEEE International Conference on Computer Vision (ICCV'05), pp. 1800-1807, October 2005.

[13] B. Leibe, A. Leonardis, and B. Schiele, "Combined object categorization and segmentation with an implicit shape model," in Proceedings of Workshop on Statistical Learning in Computer Vision, 2004.

[14] S. Lazebnik, C. Schmid, and J. Ponce, "Beyond bags of features: spatial pyramid matching for recognizing natural scene categories," in Proceedings of the IEEE Computer Society Conference on Computer Vision and Pattern Recognition (CVPR '06), vol. 2, pp. 2169-2178, New York, NY, USA, June 2006. 
[15] A. Agarwal and B. Triggs, "Hyperfeatures-multilevel local coding for visual recognition," in Proceedings of the 9th European Conference on Computer Vision (ECCV '06), vol. 3951 of Lecture Notes in Computer Science, pp. 30-43, Graz, Austria, May 2006.

[16] J. Mutch and D. G. Lowe, "Multiclass object recognition with sparse, localized features," in Proceedings of IEEE Computer Society Conference on Computer Vision and Pattern Recognition (CVPR '06), pp. 11-18, June 2006.

[17] H. Zhang, A. C. Berg, M. Maire, and J. Malik, "SVM$\mathrm{KNN}$ : discriminative nearest neighbor classification for visual category recognition," in Proceedings of the IEEE Computer Society Conference on Computer Vision and Pattern Recognition (CVPR '06), vol. 2, pp. 2126-2136, New York, NY, USA, June 2006.

[18] M. Varma and D. Ray, "Learning the discriminative powerinvariance trade-off," in Proceedings of the 11th IEEE International Conference on Computer Vision (ICCV '07), pp. 1-8, October 2007.

[19] F. Perronnin, C. Dance, G. Csurka, and M. Bressan, "Adapted vocabularies for generic visual categorization," in Proceedings of the 9th European Conference on Computer Vision (ECCV '06), vol. 3954 of Lecture Notes in Computer Science, pp. 464475, Graz, Austria, May 2006.

[20] W. Lei, "Toward a discriminative codebook: codeword selection across multi-resolution," in Proceedings of the IEEE Computer Society Conference on Computer Vision and Pattern Recognition (CVPR '07), pp. 1-8, Minneapolis, Minn, USA, June 2007.

[21] T. Yeh, J. Lee, and T. Darrell, "Adaptive vocabulary forests br dynamic indexing and category learming," in Proceedings of the 11th IEEE International Conference on Computer Vision (ICCV '07), pp. 1-8, October 2007.

[22] J. C. van Gemert, J.-M. Geusebroek, C. J. Veenman, and A. W. M. Smeulders, "Kernel codebooks for scene categorization," in Proceedings of the 10th European Conference on Computer Vision (ECCV '08), vol. 5304 of Lecture Notes in Computer Science, pp. 696-709, 2008.

[23] W. Zhang, A. Surve, X. Fern, and T. Dietterich, "Learning non-redundant codebooks for classifying complex objects," in Proceedings of the 26th International Conference on Machine Learning (ICML '09), pp. 1241-1248, June 2009.

[24] K. Grill-Spector and N. Kanwisher, "Visual recognition: as soon as you know it is there, you know what it is," Psychological Science, vol. 16, no. 2, pp. 152-160, 2005.

[25] G. Dorkó and C. Schmid, "Selection of scale-invariant parts for object class recognition," in Proceedings of the 9th IEEE International Conference on Computer Vision, pp. 634-640, October 2003.

[26] A. Opelt, A. Pinz, M. Fussenegger, and P. Auer, "Generic object recognition with boosting," IEEE Transactions on Pattern Analysis and Machine Intelligence, vol. 28, no. 3, pp. 416-431, 2006.

[27] B. Leibe, A. Leonardis, and B. Schiele, "Robust object detection with interleaved categorization and segmentation," International Journal of Computer Vision, vol. 77, no. 1-3, pp. 259289, 2008.

[28] S. Lazebnik, C. Schmid, and J. Ponce, "A maximum entropy framework for part-based texture and object recognition," in Proceedings of the 10th IEEE International Conference on Computer Vision (ICCV '05), pp. 832-838, Beijing, China, October 2005.

[29] A. Opelt, A. Pinz, and A. Zisserman, "A boundary-fragmentmodel for object detection," in Proceedings of the 9th European
Conference on Computer Vision (ECCV '06), vol. 3952 of Lecture Notes in Computer Science, pp. 575-588, Graz, Austria, May 2006.

[30] J. Shotton, A. Blake, and R. Cipolla, "Contour-based learning for object detection," in Proceedings of the 10th IEEE International Conference on Computer Vision (ICCV'05), pp. 503-510, October 2005.

[31] J. Shotton, A. Blake, and R. Cipolla, "Multiscale categorical object recognition using contour fragments," IEEE Transactions on Pattern Analysis and Machine Intelligence, vol. 30, no. 7, pp. 1270-1281, 2008.

[32] E. Hayman, B. Caputo, M. Fritz, and J.-O. Eklundh, "On the significance of real-world conditions for material classification," in Proceedings of European Conference on Computer Vision, vol. 3024 of Lecture Notes in Computer Science, pp. 253266, 2004.

[33] J. Winn and J. Shotton, "The layout consistent random field for recognizing and segmenting partially occluded objects," in Proceedings of the IEEE Computer Society Conference on Computer Vision and Pattern Recognition (CVPR '06), vol. 1, pp. 37-44, New York, NY, USA, June 2006.

[34] J. Shotton, J. Winn, C. Rother, and A. Criminisi, “TextonBoost for image understanding: multi-class object recognition and segmentation by jointly modeling texture, layout, and context," International Journal of Computer Vision, vol. 81, no. 1, pp. 2-23, 2009.

[35] P. F. Felzenszwalb, R. B. Girshick, D. McAllester, and D. Ramanan, "Object detection with discriminatively trained part-based models," IEEE Transactions on Pattern Analysis and Machine Intelligence, vol. 32, no. 9, pp. 1627-1645, 2010.

[36] V. Bruce, P. Green, and M. Georgeson, Visual Perception: Physiology, Psychology and Ecology, Psychology Press, 1995.

[37] A. Artale, E. Franconi, N. Guarino, and L. Pazzi, "Part-whole relations in object-centered systems: an overview," Data and Knowledge Engineering, vol. 20, no. 3, pp. 347-383, 1996.

[38] R. VanRullen, "Visual saliency and spike timing in the ventral visual pathway," Journal of Physiology Paris, vol. 97, no. 2-3, pp. 365-377, 2003.

[39] M. Bar, "Visual objects in context," Nature Reviews Neuroscience, vol. 5, no. 8, pp. 617-629, 2004.

[40] D. Marr, Vision: A Computational Investigation into the Human Representation and Processing of Visual Information, Henry Holt, New York, NY, USA, 1982.

[41] R. VanRullen and S. J. Thorpe, "Is it a bird? Is it a plane? Ultrarapid visual categorisation of natural and artifactual objects," Perception, vol. 30, no. 6, pp. 655-668, 2001.

[42] T. Serre, L. Wolf, S. Bileschi, M. Riesenhuber, and T. Poggio, "Robust object recognition with cortex-like mechanisms," IEEE Transactions on Pattern Analysis and Machine Intelligence, vol. 29, no. 3, pp. 411-426, 2007.

[43] N. Pinto, D. D. Cox, and J. J. DiCarlo, "Why is real-world visual object recognition hard?” PLoS Computational Biology, vol. 4, no. 1, pp. 0151-0156, 2008.

[44] R. Desimone and J. Duncan, "Neural mechanisms of selective visual attention," Annual Review of Neuroscience, vol. 18, pp. 193-222, 1995.

[45] M. Usher and E. Niebur, "Modeling the temporal dynamics of IT neurons in visual search: a mechanism for top-down selective attention," Journal of Cognitive Neuroscience, vol. 8, no. 4, pp. 311-327, 1996.

[46] G. Deco and T. S. Lee, "A unified model of spatial and object attention based on inter-cortical biased competition," Neurocomputing, vol. 44-46, pp. 775-781, 2002. 
[47] M. I. Jordan, Ed., Learning in Graphical Models, MIT Press, Cambridge, Mass, USA, 1999.

[48] F. Jurie and B. Triggs, "Creating efficient codebooks for visual recognition," in Proceedings of the 10th IEEE International Conference on Computer Vision (ICCV '05), vol. 1, pp. 604610, Beijing, China, October 2005.

[49] O. R. Joubert, G. A. Rousselet, M. Fabre-Thorpe, and D. Fize, "Rapid visual categorization of natural scene contexts with equalized amplitude spectrum and increasing phase noise," Journal of Vision, vol. 9, no. 1, pp. 1-16, 2009.

[50] W. R. Gilks, Markov Chain Monte Carlo in Practice, Chapman \& Hall/CRC, Boca Raton, Fla, USA, 1995.

[51] P. Viola and M. J. Jones, "Robust real-time face detection," International Journal of Computer Vision, vol. 57, no. 2, pp. 137-154, 2004.

[52] D. Comaniciu and P. Meer, "Mean shift: a robust approach toward feature space analysis," IEEE Transactions on Pattern Analysis and Machine Intelligence, vol. 24, no. 5, pp. 603-619, 2002.

[53] S. Agarwal and D. Roth, "Learning a sparse representation for object detection," in Proceedings of the European Conference on Computer Vision (ECCV'02), pp. 113-130, 2002.

[54] J. Willamowski, D. Arregui, G. Csurka, C. Dance, and L. Fan, "Categorizing nine visual classes using local appearance descriptors," in Proceedings of Workshop Learning for Adaptable Visual Systems Cambridge (ICPR '04), 2004.

[55] M. Weber, M. Welling, and P. Perona, "Unsupervised learning of models for recognition," in Proceedings of European Conference on Computer Vision, pp. 18-32, 2000. 